\title{
On the applicability and solution of bilevel optimization models in transportation science: A study on the existence, stability and computation of optimal solutions to stochastic mathematical programs with equilibrium constraints
}

\author{
Michael Patriksson*
}

7th May 2008

\begin{abstract}
Bilevel optimization models, and more generally MPEC (mathematical program with equilibrium constraints) models, constitute important modelling tools in transportation science and network games, as they place the classic "what-if" analysis in a proper mathematical framework. The MPEC model is also becoming a standard for the computation of optimal design solutions, where "design" may include either or both of network infrastructure investments and various types of tolls. At the same time, it does normally not sufficiently well take into account possible uncertainties and/or perturbations in problem data (travel costs and demands), and thus may not a priori guarantee robust designs under varying conditions. We consider natural stochastic extensions to a class of MPEC traffic models which explicitly incorporate data uncertainty. In stochastic programming terminology, we consider "here-and-now" models where decisions on the design must be made before observing the uncertain parameter values and the responses of the network users, and the design is chosen to minimize the expectation of the upper-level objective function. Such a model could, for example, be used to derive a fixed link pricing scheme that provides the best revenue for a given network over a given time period, where the varying traffic conditions are described by distributions of parameters in the link travel time and OD demand functions.

For a general such SMPEC network model we establish not only the existence of optimal solutions, but in particular their stability to perturbations in the probability distribution. We also provide convergence results for general algorithmic schemes based on the penalization of the equilibrium conditions or possible joint upper-level constraints, as well as for algorithms based on the discretization of the probability distribution, the latter enabling the utilization of standard MPEC algorithms. Especially the latter part utilizes relations between the traffic application of SMPEC and stochastic structural topology optimization problems.

Keywords: traffic equilibrium, communication networks, stochastic programming, stochastic mathematical program with equilibrium constraints, network design, solution stability, penalization, discretization, Monte Carlo simulation, sample average approximation.
\end{abstract}

\section{Introduction and motivation}

\subsection{Motivation}

When addressing network design and pricing problems under user equilibrium, or any kind of traffic assignment model for that matter, in the form of a hierarchical (that is, bilevel) optimization model (or, mathematical programming problem with equilibrium constraints, MPEC) it is almost always understood that data is certain or that uncertain data can be given some deterministic representation, such as a mean value. It is at the same time understood by all who develop or utilize traffic equilibrium or assignment models, that this assumption is rather simplistic. One might take the extreme approach that hierarchical models based on simple deterministic and static assignment models therefore are of little use, and that position would most likely not change even if the underlying static traffic model would be replaced by a dynamic model. Replacing a deterministic traffic equilibrium model by a stochastic one does not really

\footnotetext{
${ }^{*}$ Department of Mathematical Sciences, Chalmers University of Technology, and Mathematical Sciences, Gothenburg University, SE-41296 Göteborg, Sweden. E-mail: mipat@math.chalmers.se
} 
solve the problem either, because at least the classic ones (logit and probit, and the latter's modern relatives) also produce unique equilibrium solutions in general; hence the mapping from data to solution is not stochastic even in such models.

Since uncertainty in data is prevalent in traffic assignment models (for discussions regarding the demand function, see, e.g., Yang et al. 1991, Flyvbjerg et al. 2005, Yang et al. 2005; and Chen et al. 2006); discussions on proper travel cost/time functions are too numerous to mention, but a few basic references are Outram \& Thompson (1978), Akçelik (1991), and Uchida \& Iida (1993), and because bilevel models provide such an attractive modelling approach, there is a need for a bilevel model that can take into account the uncertainty in data.

One possibility is to use a classic 'multi-load' approach, taken from the engineering sciences, in which several representative scenarios are included through an averaging in the upper-level objective function. Structural optimization is the science of producing structures that carry loads optimally. Variations in, for example, loading conditions are often taken into account such that the effectiveness of a design is defined as the average value of the effectiveness of several 'load cases'. Because of the anticipated fact that the 'real' probability model is never known, and the reported high sensitivity of solutions to stochastic structural optimization problems with respect to small changes in the probability measure (Ben-Haim \& Elishakoff 1990, pp. 20-22), many probability-free worst-case ('pessimistic') models of uncertainty have been developed as alternatives to probabilistic ones. In such worst-case models, uncertain parameters are assumed to vary in convex sets (see further below). An efficient numerical approach to solve certain convex problems of this type is known (Ben-Tal \& Nemirovski 1997), but it has considerable drawbacks, the most serious in our context being that the uncertain data must lie in some small ellipsoid around the primal data values, which of course reduces the generality of the algorithm substantially.

This paper seeks to extend the scope of bilevel traffic models to take into account data variations in the form of a stochastic bilevel model, or SMPEC. In order to do so we must consider complications that do not arise in standard bilevel traffic models: SMPEC models are infinite-dimensional, so even the existence of optimal solutions is non-trivial to establish. The main focus of our study of the SMPEC traffic model of this paper is however one that, to some degree, can be used to validate the use of MPEC models in network design applications and in particular multi-load approaches and discretizations: we wish to establish conditions under which optimal designs change continuously with the probability distribution. We say that such solutions are stable, and the model therefore is 'robust' in some sense. Establishing the robustness of the stochastic bilevel model to be developed in this paper is also an alternative to the approach taken in some papers, where robust models are produced through reformulation (see below).

This work is built on a talk at the Royal Society discussion meeting "Networks: modelling and control" held in London 24-25 September 2007, and on the corresponding discussion paper (Patriksson 2008); the current work however is much more complete, including proofs of all the theorems stated (some of which are also new) as well as including a more thorough section on further research and a more complete bibliography, compared to the afore-mentioned discussion paper.

\subsection{Stochastic programming and robust optimization}

The problem of data uncertainty has been recognized as important for quite some time in the operations research community. The most well-known technique for dealing with uncertainty is stochastic programming (e.g., Van Slyke \& Wets 1969; Rockafellar \& Wets 1976; Kall \& Wallace 1994; and Birge \& Louveaux 1997). Typically, one wants to minimize the expected cost of decisions, that must be made without the complete knowledge of data. In 'here-and-now' models decisions are made once, but in certain types of applications decisions can be made at one or several future recourse stages. In the latter case, the here-and-now decision represents the first-stage decision which is made to properly hedge against future outcomes of the uncertain data. In these types of problems one assumes that the distribution of uncertainty is known, or, at least, can be well approximated.

The term 'robust optimization' (RO) has been and is used in a variety of contexts. An important aspect in most applications is the assumption that the distribution of data is known to be confined to certain (typically bounded) 'uncertainty sets'. For particular problem types, such as linear programming (e.g., Soyster 1973; Ben-Tal \& Nemirovski 1999, 2002), convex quadratic programming (e.g., El Ghaoui \& Lebret 1997), and certain maximum-stiffness truss topology optimization problems (e.g., Ben-Tal \& Nemirovski 1997, Ben-Tal et al. 1999), and particular uncertainty sets, RO offers computationally tractable (i.e., polynomially solvable) robust versions. Because feasibility is required for every realization of the data, the robust counterparts include a semi-infinite system of constraints (whence tractability is 
only possible to obtain in the above types of instances). Importantly, it is at the same time a worst-case modelling approach.

Through the above modelling technique one imposes robustness on a model. Our main interest is to investigate the robustness of SMPEC models, particularly in transportation science and network games, in the hope that a reformulation is not necessary. As we shall see, it is in some circumstances possible to establish the robustness of an SMPEC model, but reformulations may be necessary in some cases. SMPEC models, and indeed also MPEC models, are normally not tractable; robust reformulation will therefore tend not to be tractable either.

\subsection{Stochastic mathematical programs with equilibrium constraints}

A mathematical program with equilibrium constraints (MPEC) is an optimization problem with two types of variables: primary ones, or 'design' variables, and secondary variables, or 'responses'. The responses are evaluated as part of the constraints of the problem, often in the form of a secondary optimization problem (then often referred to as the 'lower-level problem'). The overall objective is optimized according to a criterion in terms of both design and response.

In order to represent this common framework in mathematical notation, suppose the design is represented by a vector $x \in \mathbb{R}^{n}$ and the response by a vector $y \in \mathbb{R}^{m}$. If the objective is to minimize the function $f: \mathbb{R}^{n} \times \mathbb{R}^{m} \rightarrow \mathbb{R}$ and the response can be described by the point-to-set mapping $S: \mathbb{R}^{n} \rightrightarrows \mathbb{R}^{m}$, then we can describe the MPEC problem as that to

$$
(\text { MPEC })\left\{\begin{aligned}
\underset{(x, y)}{\operatorname{minimize}} f(x, y) & , \\
\text { subject to }(x, y) & \in Z, \\
y & \in S(x),
\end{aligned}\right.
$$

where $Z \subseteq \mathbb{R}^{n} \times \mathbb{R}^{m}$ is the set of admissible designs and responses.

If the mapping $x \mapsto S(x)$ describes the solution of a variational inequality (VI), parameterized by $x$, then the inclusion $y \in S(x)$ holds if and only if

$$
-F(x, y) \in N_{Y(x)}(y)
$$

where $Y(x) \subseteq \mathbb{R}^{m}$ is the feasible set of the VI, $F(x, \cdot): \mathbb{R}^{m} \rightarrow \mathbb{R}^{m}$ is the VI cost mapping, and $N_{Y(x)}: Y(x) \rightrightarrows \mathbb{R}^{m}$ is the normal cone mapping associated with the set $Y(x)$, all parameterized by $x$. We assume throughout that the set $Y(x)$ is closed and convex, whence its normal cone is the standard one in convex analysis: for $y \notin Y(x), N_{Y(x)}(y):=\emptyset$, and for $y \in Y(x), N_{Y(x)}(y):=\left\{z \in \mathbb{R}^{m} \mid z^{\mathrm{T}}(w-y) \leq\right.$ $0, w \in Y(x)\}$. If $F(x, \cdot)=\nabla \varphi(x, \cdot)$ for some $C^{1}$ function $\varphi(x, \cdot): \mathbb{R}^{m} \rightarrow \mathbb{R}$ then (1) is the first-order optimality conditions for the parameterized optimization problem to

$$
\underset{y \in Y(x)}{\operatorname{minimize}} \varphi(x, y) \text {. }
$$

The mapping $x \mapsto S(x)$ could also be associated with KKT conditions for an optimization problem like (2), and hence also would include explicit Lagrange multipliers. For general overviews of MPEC and VI models and their analysis, see Luo et al. (1996), Bard (1998) and Outrata et al. (1998), and Facchinei \& Pang (2003a,b), respectively.

The existence of optimal solutions to the problem (MPEC) follows under standard assumptions that hark back to a version of Weierstrass' Theorem: if the set of feasible solutions to (MPEC) is non-empty and closed, and the objective function is lower semi-continuous ${ }^{1}$ and weakly coercive ${ }^{2}$ (this combination of properties is called 'inf-compact'), then there exists a closed and bounded set of optimal solutions (see, e.g., Zhang 1994). In particular, closedness of the feasible set follows in general from a closedness assumption on the mapping $S$ and of the set $Z$. In the examples to follow, generally stronger properties still will hold. In particular, for reasons of computational ease and also for a well-defined optimal value one normally imposes conditions on the lower-level problem such that it is unique. The mapping $x \mapsto S(x)$

\footnotetext{
${ }^{1}$ Suppose $S \subseteq \mathbb{R}^{n}$ is non-empty and closed. A function $f: S \rightarrow \mathbb{R}$ is said to be lower semi-continuous on $S$ if for every $x \in S$ it holds that if $x_{k} \rightarrow x$ then $f(x) \leq \liminf _{k \rightarrow \infty} f\left(x_{k}\right)$. In other words, any discontinuous jumps are downward ones. Equivalently, all level sets of $f$ are closed sets.

${ }^{2}$ Again let $S \subseteq \mathbb{R}^{n}$ be non-empty and closed. A function $f: S \rightarrow \mathbb{R}$ is said to be weakly coercive with respect to $S$ if either $S$ is bounded or $\lim _{\substack{\| x \in S \\ x \in S}} f(x)=\infty$ holds. Equivalently, all level sets of $f$ on $S$ are bounded.
} 
then is single-valued, and for a given design $x$, the objective value equals $f(x, y(x))$, where $\{y(x)\}=S(x)$. In order to be able to utilize descent methods one often also imposes still stronger conditions, such that the upper-level objective function $x \mapsto f(x, S(x))$ is (locally) Lipschitz continuous; in these circumstances one can calculate and hence utilize subgradients of $f$.

Notice that efficient computations require that the structure of $S$ is known; in other words, normally it is required of the mapping $S$ that it is defined as the solution of an explicit system, as in the case of the parameterized VI (1) or the parameterized optimization problem (2); otherwise we face a problem that might be referred to as a 'black box' optimization problem. (Such problems often arise in optimization problems where a simulation is part of the objective function evaluation; the SMPEC model could certainly be an appropriate modelling framework also for such problems, but only under the abovementioned requirement that something is known about the structure of the simulation problem and the associated uncertainties.)

We focus here on traffic and communication networks, whence $x$ is the vector of design parameters, while the mapping $S$ describes the solution to a traffic equilibrium (or, assignment) type model having $y$ as the corresponding equilibrium traffic flows and (dis)utilities. The set $Z$ could represent constraints on the available investments (or, tolls, etcetera), which then are independent on the response $y$; it could also represent maximum allowed travel times (e.g., Abdulaal \& LeBlanc 1979; Marcotte 1986; Friesz et al. 1992; Marcotte \& Marquis 1992; Cree et al. 1998; Meng et al. 2001; Chiou 2005; and Josefsson \& Patriksson 2007), exhaust emissions, or constraints defined by equity measures in a multi-class model (e.g., Meng \& Yang 2002, Eliasson \& Mattsson 2006, and Stewart 2007), or equilibrium travel times (e.g., Chen et al. 2002 and Sumalee et al. 2006), which are examples of joint upper-level constraints.

The subject of rate control in computer communication networks is also strongly related to congestion control in traffic networks, although the intrinsic existence of link flow capacity constraints in the former enforces the need to rephrase the Wardrop principle (cf. Larsson \& Patriksson 1994b). Ralph (2008) establishes the equivalence of rate flow equilibria for a model of the transmission rate protocol (TCP; see Kelly et al. 1998; and Kelly \& Voice 2005) to a mixed complementarity problem; various design and control problem of the MPEC type are then discussed. Robust such models that determine good transmission rates in the light of uncertainties in, e.g., link capacities, in the form of SMPECs are very natural extensions to study in the future, as are the corresponding SMPECs that are based on side constrained traffic equilibria (Larsson \& Patriksson 1994b, 1998, 1999).

We have already and will also in the following discuss in brief certain applications to structural (topology) optimization, wherein $x$ refers to design variables describing an amount of material (thicknesses or volumes), while $y$ refers to an equilibrium state (stresses and displacements); the equilibrium, described by the mapping $S$, corresponds to an energy minimization problem, parameterized by $x$. The set $Z$ represents possible constraints on available material and/or on the (maximum) stresses. For an overview of structural (topology) optimization, see Bendsøe \& Sigmund (2003).

Our focus is on the analysis of a stochastic extension of the MPEC problem. It is known in the literature as stochastic MPEC, or SMPEC, a term coined in the paper by Patriksson \& Wynter (1999). We suppose that $(\Omega, \Theta, \mathrm{P})$ is a complete probability space. When considering the space $\mathbb{R}^{n} \times \Omega$ we equip it always with the sigma algebra given by the product of the Borel sigma algebra of $\mathbb{R}^{n}$ and $\Theta$.

The SMPEC problem then is that to

$$
\left(\operatorname{SMPEC}_{\Omega}\right)\left\{\begin{aligned}
\underset{(x, y(\cdot))}{\operatorname{minimize}} \mathrm{E}_{\omega}[f(x, y(\omega), \omega)]:= & \int_{\Omega} f(x, y(\omega), \omega) \mathrm{P}(d \omega), \\
\text { subject to }(x, y(\omega)) \in Z(\omega), & \text { P-a.s. } \\
y(\omega) \in S(x, \omega), & \text { P-a.s. }
\end{aligned}\right.
$$

where $\mathrm{E}_{\omega}$ denotes expected value, $y: \Omega \rightarrow \mathbb{R}^{m}$ is a random element of the probability space $(\Omega, \Theta, \mathrm{P})$, $Z: \Omega \rightrightarrows \mathbb{R}^{n} \times \mathbb{R}^{m}$ is a point-to-set mapping representing the upper-level constraints, and $S: \mathbb{R}^{n} \times \Omega \rightrightarrows \mathbb{R}^{m}$ defines the set of solutions to the lower-level parametric variational inequality problem,

$$
S(x, \omega):=\left\{y \in \mathbb{R}^{m} \mid-F(x, y, \omega) \in N_{Y(x, \omega)}(y)\right\} .
$$

The above lower-level problem is defined by a mapping $F: \mathbb{R}^{n} \times \mathbb{R}^{m} \times \Omega \rightarrow \mathbb{R}^{m}$ and a feasible set mapping $Y: \mathbb{R}^{n} \times \Omega \rightarrow \mathbb{R}^{m}$ having closed convex images with $N_{Y(x, \omega)}: \mathbb{R}^{m} \rightrightarrows \mathbb{R}^{m}$ denoting the normal cone mapping to the set $Y(x, \omega)$.

The problem $\left(\mathrm{SMPEC}_{\Omega}\right)$ represents a generalization of the problem (MPEC) wherein we explicitly incorporate uncertainties in the problem data to obtain robust solution. Uncertainties are here taken 
into account in the minimization of the expectation of the objective values over the uncertainty set. Alternative approaches, such as worst-case, or maximum regret, models are discussed briefly below.

It should be noted that the stochastic formulation $\left(\mathrm{SMPEC}_{\Omega}\right)$ is very different from the deterministic problem where the optimization is performed over $x$ given an average (or, mean) scenario over the uncertainty space; a simple example in Gürkan et al. (1999b) illustrates this fact clearly; see also Wallace (2000) for comments on the inappropriate use of what-if analyses to a truly stochastic problem.

As for the case of the problem (MPEC) we must decide on how to interpret the objective value of $\left(\operatorname{SMPEC}_{\Omega}\right)$ when the lower-level solution is not unique for given $x$ and $\omega$; as is the tradition for (MPEC), we will assume that the choice of solution $y$ is the one that minimizes the objective value for given $x$ and $\omega$; this explains the notation underneath the minimization operator in $\left(\mathrm{SMPEC}_{\Omega}\right)$.

A forerunner to the paper by Patriksson \& Wynter (1999) was the conference presentation "Bilevel stochastic programming for network equilibrium problems" at the International Symposium on Mathematical Programming (ISMP97) by Wynter (1997) (see also Wynter 2002), in which the combination of stochastic programming and bilevel optimization was discussed in the context of two network planning problems: a toll setting problem under user equilibrium and a pricing problem in a spatial-price equilibrium setting, where in both cases demand is stochastic. In that presentation stochasticity enters in a discretized manner. The paper by Patriksson \& Wynter (1999) analyzes continuously distributed cases of SMPEC but discusses stochastic bilevel (Stackelberg) models only in principle.

The first published real application of SMPEC where the distribution is continuous was topology optimization models in structural mechanics. In Christiansen et al. (2001) and Patriksson \& Petersson (2002) the existence and computation of discretized versions of topology optimization models were analyzed, while Evgrafov et al. (2002, 2003) analyzed the continuously distributed case. The paper by Evgrafov \& Patriksson (2004) provides the first existence analysis for general SMPEC models as well as an inexact penalty method, while Evgrafov \& Patriksson (2003a,b) investigated stability issues in the context of topology optimization; in particular, the latter two references established that in general the optimal design cannot be expected to vary continuously with the probability measure, but that continuity indeed could be established in some special circumstances for convex problems. We shall in this paper provide a corresponding analysis in the context of optimal design in networks, and reach stronger results in that they do not impose convexity on the model.

Other applications of special cases of SMPEC include Stackelberg-Nash-Cournot equilibrium problems (De Wolf \& Smeers 1997; Xu 2005), in which case the lower-level problem is a nonlinear complementarity problem (see also the recent extension in Hobbs \& Pang 2007); the special SMPEC model is then referred to as SMPCC (stochastic program with complementarity constraints) which is characterized by the presence of a nonlinear complementarity system. Further technical developments of SMPEC include the work of Shapiro (2006), which in particular studies the measurability and continuity properties of the integrand functions involved. The paper also studies numerical approaches for cases of the problem where the lower-level problem is a special generalized equation, in particular Monte Carlo sampling approaches. The resulting sample average approximation algorithm is also studied in detail in Shapiro \& Xu (2005) for a special SMPCC model, as well as in Shapiro (2008) for a multitude of stochastic problems. The present paper offers as well a summary analysis of the application of discretization approaches to SMPEC models in the context of traffic networks.

\subsection{Outline}

The next section provides an existence result for the problem $\left(\mathrm{SMPEC}_{\Omega}\right)$, taken from Evgrafov \& Patriksson (2004, Theorem 2.1), as well as some additional discussions on problem interpretations. Section 3 then introduces our traffic SMPEC model. The lower-level problem is a standard (possibly asymmetric) deterministic traffic equilibrium model; the technical results that then follow hold also if the SMPEC model is built upon a variety of stochastic traffic equilibrium models (see further Section 6 on this issue). For future use, we introduce two versions of the hierarchical model: the first one allows for joint upper-level constraints, that is, simultaneous constraints in the designs $x$ and the responses (lower-level solutions) $y$, while the second model has upper-level constraints only in $x$. The reason for this has already been alluded to in the context of our discussion on structural topology optimization: some results are in general not possible to obtain for the first type of model.

Section 4 establishes the main result of this paper: that in the absence of joint upper-level constraints the optimal designs are continuous in the probability distribution; hence, we can conclude that optimal design solutions are stable, and the SMPEC traffic model is robust. Section 5 establishes the convergence 
of solution approaches to the SMPEC problem at hand. The first is a classic inexact exterior penalty method in which either the lower-level problem or any joint upper-level constraints are penalized. The second is a natural approach for the continuously distributed problem $\left(\mathrm{SMPEC}_{\Omega}\right)$ : we consider two related discretization approaches; the first is based on the method of mechanical quadratures, while the second is a straightforward application of sample average approximation. A final section discusses some interesting future research topics.

\section{Existence of optimal solutions}

Let $X$ denote the projection of the feasible set of the problem $\left(\mathrm{SMPEC}_{\Omega}\right)$ onto the space of $x$ variables:

$$
X:=\left\{x \in \mathbb{R}^{n} \mid \exists y(\omega):(x, y(\omega)) \in Z(\omega) \text { for almost every } \omega\right\},
$$

and let

$$
Z_{x}(\omega):=\left\{y \in \mathbb{R}^{m} \mid(x, y) \in Z(\omega)\right\} .
$$

Let us also denote by $\Upsilon_{x}(\omega)$ the $x$-slice of the feasible set of $\left(\mathrm{SMPEC}_{\Omega}\right)$,

$$
\Upsilon_{x}(\omega):=Z_{x}(\omega) \cap S(x, \omega) .
$$

The existence result is based on the measurability in $\omega$ for fixed $x$ of this mapping. (A closed valued mapping $A: \Omega \rightrightarrows \mathbb{R}^{n}$ is said to be measurable if $A^{-1}(V) \in \Theta$ for every closed set $V \subset \mathbb{R}^{n}$, cf. Himmelberg 1975, Section 2; Castaing \& Valadier 1977, Chapter III; Aubin \& Frankowska 1990, Chapter 8; or Rockafellar \& Wets 1998, Chapter 14.) We shall assume throughout that $Z_{x}(\omega)$ and $Y(x, \omega)$ are measurable in $\omega$ for every $x \in \mathbb{R}^{n}$; notice that this continuity type of assumption is not very restrictive.

According to Evgrafov \& Patriksson (2004, Lemma 2.1) (see also Rockafellar \& Wets 1998, Theorem 14.26 and Example 14.15(b)), if in addition $F$ is continuous in $y$ and measurable in $\omega$ (which is referred to as the 'Carathéodory property') for every $x$, and $Y$ has closed convex images for every $x$ and almost every $\omega$, then the mapping $S$ is measurable in $\omega$ for every $x$. Results such as Rockafellar \& Wets (1998, Theorem 14.37) refer to the measurability of solution mappings of parameterized optimization problems rather than of solutions to the more general variational inequality problems that we are concerned with, but as remarked in Shapiro (2006) variational inequalities can be transformed to optimization problems through the use of merit (or, gap) functions (e.g., Auchmuty 1989; Fukushima 1992; Larsson \& Patriksson 1994a; and Facchinei \& Pang 2003a), to which Theorem 14.37 in Rockafellar \& Wets (1998), for example, can be applied. Thanks to the measurability of $S$ and the above assumptions it then follows that the integrand function is measurable as well.

The following result, quoted from Evgrafov \& Patriksson (2004, Theorem 2.1), establishes the existence of optimal solutions to the problem $\left(\mathrm{SMPEC}_{\Omega}\right)$. This theorem, in turn, generalizes existence results for (MPEC) in a natural manner.

THEOREM 1 (existence of optimal solutions) Suppose that the following assumptions are fulfilled: the mappings $Z_{x}(\cdot)$ and $S(x, \cdot)$ are measurable for every $x$; the set $Z(\omega)$ and the mapping $x \mapsto S(x, \omega)$ are closed for almost every $\omega \in \Omega$ and every $x$; the function $f$ is continuous in $(x, y)$, measurable in $\omega$, uniformly weakly coercive with respect to $x$ over the set $X,{ }^{3}$ and bounded from below by a $(\Theta, P)$-integrable function; for every $x \in X$, there is a neighborhood $U_{x} \ni x$ such that the set $\cup_{\bar{x} \in U_{x}} Z_{\bar{x}}(\omega)$ is bounded for almost every $\omega \in \Omega$; and the set $\Upsilon_{x_{0}}(\omega)$ is nonempty for some $x_{0} \in X$ and almost every $\omega \in \Omega$. Then, the problem $\left(\mathrm{SMPEC}_{\Omega}\right)$ has at least one optimal solution.

Clearly, the case of joint upper-level constraints is a complicating factor, and we shall see later that robustness results and computations are more easily discussed in a framework where $Z$ is independent of $y$. A corollary to the above theorem illustrates how the analysis then simplifies.

Corollary 2 (existence of optimal solutions) Suppose that the upper-level feasible set $Z$ is independent of $y$, and hence the upper-level constraints can be written as $x \in X \subseteq \mathbb{R}^{n}$. Then, the assumptions of the theorem can be replaced by the following: the mapping $S(x, \cdot)$ is measurable for every $x$; the set $X$ is closed, and the mapping $x \mapsto S(x, \omega)$ is closed for almost every $\omega \in \Omega$; the function $f$ is continuous in $(x, y)$, measurable in $\omega$, uniformly weakly coercive with respect to $x$ over the set $X$, and bounded from below by a $(\Theta, P)$-integrable function; the set $S\left(x_{0}, \omega\right)$ is nonempty for some $x_{0} \in X$ and almost every $\omega \in \Omega$.

\footnotetext{
${ }^{3}$ That is, that the set $\left\{x \in X \mid \exists y \in \mathbb{R}^{m}, \omega \in \Omega\right.$ such that $\left.f(x, y, \omega) \leq c\right\}$ is bounded for every $c \in \mathbb{R}$.
} 
In this latter case where the upper-level constraints are defined only in terms of $x$, we can provide a simple interpretation of the problem $\left(\mathrm{SMPEC}_{\Omega}\right)$. First, we recall that we select the response $y$ for which we obtain the minimum value of the upper-level objective; we can then write the problem $\left(\mathrm{SMPEC}_{\Omega}\right)$ as the problem to

$$
\underset{x \in X ; y(\cdot) \in S(x, \cdot)}{\operatorname{minimize}} \mathrm{E}[f(x, y(\omega), \omega)],
$$

where the optimization is performed over all $x \in X$ and all measurable selections $y(\omega) \in S(x, \omega)$. Equivalently, we may write the problem as that to

$$
\underset{x \in X}{\operatorname{minimize}} w(x):=\mathrm{E}[v(x, \omega)]
$$

where

$$
v(x, \omega):=\inf _{y \in S(x, \omega)} f(x, y, \omega) .
$$

Notice that if for a given $x \in X$ the set $S(x, \omega)$ is empty with a positive probability we define $w(x):=\infty$; implicitly, therefore, the optimization over $x$ is performed such that the set $S(x, \omega)$ is non-empty for almost every $\omega \in \Omega$.

A comparison is finally made with the case of a finite support of $\Omega$. Suppose that $\Omega=\left\{\omega_{1}, \ldots, \omega_{K}\right\}$ is finite with probabilities $p_{1}, \ldots, p_{K}$ (satisfying $p_{i} \geq 0$ for $i=1, \ldots, K$ and $\sum_{i=1}^{K} p_{i}=1$ ). Then we may write the problem (4) as the problem to

$$
\left(\mathrm{SMPEC}_{K}\right)\left\{\begin{array}{l}
\underset{\left(x, y_{1}, \ldots, y_{K}\right)}{\operatorname{minimize}} \sum_{k=1}^{K} p_{k} f\left(x, y_{k}, \omega_{k}\right), \\
\text { subject to } \quad x \in X, \\
\quad y_{k} \in S\left(x, \omega_{k}\right), \quad k=1, \ldots, K .
\end{array}\right.
$$

In the field of structural topology optimization this is a classic modelling approach which was discussed already in the first section. If, for example, the external load is stochastic, then $\left(\mathrm{SMPEC}_{K}\right)$ represents a 'multi-load case' model where $K$ load scenarios are incorporated into the same design optimization model, together with their probabilities, resulting in a minimum average design 'cost'. (See, e.g., Marti 1997; Patriksson \& Wynter 1999; and Christiansen et al. 2001.) Alternative modelling approaches utilize reliability constraints (e.g., Marti 1997) or robust optimization via semidefinite programming (e.g., BenTal \& Nemirovski 1997; and Ben-Tal et al. 1999). Notice that the discretized problem $\left(\mathrm{SMPEC}_{K}\right)$ is nothing but a larger-scale version of (MPEC), and can therefore, in principle, be solved by the same type of methods.

In the context of transportation science, a recent example of the statement of a simple traffic model of the form $\left(\mathrm{SMPEC}_{K}\right)$ is found in Ukkusuri \& Patil (2006), where the uncertain (fixed) demand is represented by scenarios. (The more recent paper by Ukkusuri et al. 2007, introduces an objective function incorporating both the expected total system travel cost and its standard deviation as a measure of 'robustness', and also includes binary investment variables.) We will return to this type of model later on when considering discretization approaches to the problem $\left(\mathrm{SMPEC}_{\Omega}\right)$.

\section{A representative SMPEC traffic model}

We provide a representative traffic equilibrium model, for use in the analysis of the sections that follow. While the discussion concerns traffic on roads, the same model form is of course also applicable to the analysis of (computer) communication network problems, and potentially also in other, similar, fields.

\subsection{Traffic equilibrium}

Let $\mathcal{G}=(\mathcal{N}, \mathcal{L})$ be a transportation network, where $\mathcal{N}$ and $\mathcal{L}$ are the sets of nodes and directed links, respectively. For certain ordered pairs of nodes, $(p, q) \in \mathcal{C}$, where node $p$ is an origin, node $q$ is a destination, and $\mathcal{C}$ is a subset of $\mathcal{N} \times \mathcal{N}$, there is a transport demand, which may be constant or given by a function of the travel cost. We assume that the network is strongly connected, so that at least one route joins each origin-destination (OD) pair. 
Wardrop's user equilibrium principle (Wardrop 1952; Beckmann et al. 1956; Sheffi 1985; Patriksson 1994; and Marcotte \& Patriksson 2007) states that for every OD pair $(p, q) \in \mathcal{C}$, the travel costs of the routes utilized are equal and minimal for each individual user. We denote by $\mathcal{R}_{p q}$ the set of simple (loop-free) routes for OD pair $(p, q)$, by $h_{r}$ the flow on route $r \in \mathcal{R}_{p q}$, and by $c_{r}$ the travel cost on the route as experienced by an individual user.

We introduce the design parameter $x \in \mathbb{R}^{n}$. This parameter could be present in one or both of the travel cost and demand functions. We assume that the (continuous) travel cost function has the form $c(x, \cdot): \mathbb{R}_{+}^{|\mathcal{R}|} \rightarrow \mathbb{R}^{|\mathcal{R}|}$ given a value of $x$, where $|\mathcal{R}|$ denotes the total number of routes in the network, and $\mathbb{R}_{+}:=\{x \in \mathbb{R} \mid x \geq 0\}$. Further, the demand function is given by $g(x, \cdot): \mathbb{R}^{|\mathcal{C}|} \rightarrow \mathbb{R}_{+}^{|\mathcal{C}|}$. Regarding the latter function we shall, for simplicity and familiarity, assume that either $g$ is independent of the travel cost (hence a case of a fixed demand model) or is such that $-g(x, \cdot)$ is (at least) strictly monotone for each $x$; in the latter case we can then utilize the standard VI formulation based on the inverse of $g(x, \cdot)$. (These assumptions are rather standard in the literature on traffic modelling.)

In an application to equilibrium network design, pricing and control models (e.g., Marcotte 1986; Patriksson \& Rockafellar 2002; Kelly 2006; Eliasson \& Mattsson 2006; and Josefsson \& Patriksson 2007) $n \approx|\mathcal{L}|$ may hold, while in an application to OD estimation (e.g., Gur et al. 1979; Cascetta \& Nguyen 1988; Yang 1995; and Chen \& Florian 1998) $n$ is in the order of $|\mathcal{C}|$.

We also introduce the matrix $\Gamma \in \mathbb{R}^{|\mathcal{R}| \times|\mathcal{C}|}$, which is the route-OD pair incidence matrix (i.e., the element $\gamma_{r k}$ is 1 if route $r$ joins OD pair $k=(p, q) \in \mathcal{C}$, and 0 otherwise). Then, demand-feasibility is described by the conditions that $h \in \mathbb{R}_{+}^{|\mathcal{R}|}$ and

$$
\Gamma^{\mathrm{T}} h=g(x, \pi)
$$

holds, while the Wardrop equilibrium conditions for the route flows are that

$$
\begin{array}{rlll}
h_{r}>0 & \Longrightarrow c_{r}(x, h)=\pi_{p q}, & r \in \mathcal{R}_{p q}, & (p, q) \in \mathcal{C}, \\
h_{r}=0 \Longrightarrow c_{r}(x, h) \geq \pi_{p q}, & r \in \mathcal{R}_{p q}, & (p, q) \in \mathcal{C},
\end{array}
$$

holds, where the value of $\pi_{p q}:=\pi_{p q}(x, h)$ is the minimal (i.e., equilibrium) route cost in OD pair $(p, q)$. By the non-negativity of the route flows, the system (5)-(6) can more compactly be written as the mixed complementarity problem (MCP)

$$
\begin{aligned}
0^{|\mathcal{R}|} \leq h \perp(c(x, h)-\Gamma \pi) & \geq 0^{|\mathcal{R}|}, \\
\Gamma^{\mathrm{T}} h & =g(x, \pi),
\end{aligned}
$$

where $a \perp b$, for two arbitrary vectors $a, b \in \mathbb{R}^{n}$, means that $a^{\mathrm{T}} b=0$. (By non-negativity, this implies that $a_{j} \cdot b_{j}=0$ for all $j$.)

While we here work with a link-route representation of flows there is a corresponding link-node representation, where conditions are imposed on the conservation of flows at intersections; see, e.g., Patriksson (1994) and Marcotte \& Patriksson (2007). The analysis that follows is valid without any alterations for such a problem representation.

In general, we shall need to assume that the route cost is additive. For each link $l \in \mathcal{L}$, the travel cost has the form $t_{l}(x, v)$, where $v \in \mathbb{R}^{|\mathcal{L}|}$ is the vector of link flows. The route and link travel costs and flows are related through a route-link incidence matrix, $\Lambda \in\{0,1\}^{|\mathcal{L}| \times|\mathcal{R}|}$, whose element $\lambda_{l r}$ equals one if route $r \in \mathcal{R}$ utilizes link $l \in \mathcal{L}$, and zero otherwise. Route $r$ has an additive route $\operatorname{cost} c_{r}(x, h)$ if it is the sum of the costs of using all the links defining it. In other words, $c_{r}(x, h)=\sum_{l \in \mathcal{L}} \lambda_{l r} t_{l}(x, v)$. In short, then, $c(x, h)=\Lambda^{\mathrm{T}} t(x, v)$. Also, implicit in this relationship is the assumption that the pair $(h, v)$ is consistent, in the sense that $v$ equals the sum of the route flows: $v=\Lambda h$. We shall use the representation in terms of $v$, as it is an entity for which we can introduce conditions ensuring that uniqueness holds at equilibrium. In general, we shall assume that $t$ is (at least) strictly monotone in $v$ for every $x$. Notice, however, that it is in each of the results to follow possible for the function $t$ to be only monotone for some values of $v$ for every $x$, as long as it is strictly monotone around the equilibrium solution for every $x$; thus, so called "flat spots" of the travel cost are allowed, again as long as these are not situated around an equilibrium.

A more familiar representation of the Wardrop conditions (7) is that of a variational inequality,

$$
-F(x, y) \in N_{C}(y)
$$

where $y \in \mathbb{R}^{m}, C \subseteq \mathbb{R}^{m}$ is a closed and convex set, $F(x, \cdot): C \rightarrow \mathbb{R}^{m}$ is smooth, and where the normal cone mapping is as defined in Section 1.3. 
Letting

$$
\begin{aligned}
y & :=\left(\begin{array}{c}
h \\
\pi \\
v
\end{array}\right) \in \mathbb{R}^{|\mathcal{R}|} \times \mathbb{R}^{|\mathcal{C}|} \times \mathbb{R}^{|\mathcal{L}|}, \\
F(x, y) & :=\left(\begin{array}{c}
\Lambda^{\mathrm{T}} t(x, v)-\Gamma \pi \\
\Gamma^{\mathrm{T}} h-g(x, \pi) \\
v-\Lambda h
\end{array}\right), \quad \text { and } \\
C & :=\mathbb{R}_{+}^{|\mathcal{R}|} \times \mathbb{R}^{|\mathcal{C}|} \times \mathbb{R}^{|\mathcal{L}|},
\end{aligned}
$$

we obtain an equivalent VI formulation from (7), where $F$ is parameterized by $x$. The equivalence between a nonlinear complementarity problem (NCP) [that is, (7a)] and a VI over the non-negative orthant [that is, $\left.-[c(x, h)-\Gamma \pi] \in N_{\mathbb{R}_{+}^{|\mathcal{R}|}}(h)\right]$ was established by Karamardian $(1969 \mathrm{a}, \mathrm{b})$. That $(7 \mathrm{~b})$ is equivalent to the statement that $-\left[\Gamma^{\mathrm{T}} h-g(x, \pi)\right] \in N_{\mathbb{R}|\mathcal{C}|}(\pi)$ follows trivially, since $N_{\mathbb{R}|\mathcal{C}|}$ is identically zero. Similarly, the equation $v=\Lambda h$ comes out as the last row of $(8),(9):-[v-\Lambda h] \in N_{\mathbb{R}^{\mid \mathcal{L}} \mid}(v)$. Solutions exist to the problem (8), (9) whenever $t(x, \cdot)$ is continuous and $g(x, \cdot)$ is non-negative and upper bounded by some nonnegative vector (cf., e.g., Marcotte \& Patriksson 2007, Theorem 4).

As remarked above, (9) is a mixed complementarity problem. If it can be shown that the travel costs are always positive then (9) can equivalently be transformed into a nonlinear complementarity problem (e.g., Aashtiani \& Magnanti 1981). In any case, our SMPEC model can be written in terms of an SMPCC, which in turn enables us to utilize specialized algorithms (e.g., Fukushima \& Pang 1999; Scholtes 2001; Hu \& Ralph 2004; Shapiro \& Xu 2005; Leyffer et al. 2007; and Ralph 2008).

We remarked above that the demand function is assumed to be of either of two different forms. In the case when the corresponding variational inequality models define first-order optimality conditions for an optimization problem, the two corresponding optimization formulations illustrate an important feature of the lower-level traffic equilibrium problem that we utilize heavily: its constraints do not depend on the design vector $x$; hence the equilibrium problem always exhibits feasible solutions, and according to the properties of the cost and demand functions assumed, equilibria will therefore also always exist. In the case of fixed demands, the optimization formulation is that to

$$
\begin{array}{cc}
\underset{v}{\operatorname{minimize}} & \phi(x, v):=\sum_{l \in \mathcal{L}} \int_{0}^{v_{l}} t_{l}(x, s) d s, \\
\text { subject to } & \Gamma^{\mathrm{T}} h=d, \\
& h \geq 0^{|\mathcal{R}|}, \\
v & =\Lambda h,
\end{array}
$$

while in the case of a strictly negatively monotone demand function (whence it has an inverse, denoted $\xi(x, \cdot))$, the optimization formulation is that to

$$
\begin{array}{cl}
\underset{(v, d)}{\operatorname{minimize}} & \phi(x, v, d):=\sum_{l \in \mathcal{L}} \int_{0}^{v_{l}} t_{l}(x, s) d s-\sum_{k \in \mathcal{C}} \int_{0}^{d_{k}} \xi_{k}(x, s) d s, \\
\text { subject to } & \Gamma^{\mathrm{T}} h=d, \\
& h \geq 0^{|\mathcal{R}|} \\
v & =\Lambda h .
\end{array}
$$

In order to produce a hierarchical traffic model, we introduce finally a continuous function $f: \mathbb{R}^{n} \times$ $\mathbb{R}^{m} \rightarrow \mathbb{R}$, and a possible set of jointly feasible designs and responses, $Z \subset \mathbb{R}^{n} \times \mathbb{R}^{m}$. The corresponding MPEC model then is to

$$
(\mathrm{HTM})\left\{\begin{aligned}
\underset{(x, y)}{\operatorname{minimize}} f(x, y) & , \\
\text { subject to }(x, y) & \in Z, \\
y & \in S(x),
\end{aligned}\right.
$$

where $S(x):=\left\{y \in \mathbb{R}^{m} \mid(8)\right.$, (9) holds $\}$.

The existence of solutions to the problem (HTM) follows under standard arguments, as it is an instance of (MPEC). The mapping $S$ is closed as soon as $t$ and $g$ are continuous, which we have assumed. We further assume that the set $Z$ and the function $f$ are so defined that there exist feasible solutions, and 
that $f$ is inf-compact there. The most natural assumptions under which this is guaranteed to hold is that $f$ is continuous, $t(x, \cdot)$ and $-g(x, \cdot)$ are strictly monotone, and the set $Z$ is independent of $y$ and compact; indeed, in most applications it is simply a box in $x$ corresponding to investment bounds.

\subsection{An SMPEC traffic model}

We next introduce a natural stochastic extension of this model, following the notation developed on Section 1.3. Consider the problem to

$$
\left(\operatorname{SHTM}_{\Omega}\right)\left\{\begin{array}{cc}
\underset{(x, y(\cdot))}{\operatorname{minimize}} \mathrm{E}_{\omega}[f(x, y(\omega), \omega)]:=\int_{\Omega} f(x, y(\omega), \omega) \mathrm{P}(d \omega), \\
\text { subject to }(x, y(\omega)) \in Z(\omega), \quad \text { P-a.s. } \\
y(\omega) \in S(x, \omega), \quad \text { P-a.s. }
\end{array}\right.
$$

where $y: \Omega \rightarrow \mathbb{R}^{m}$ is a random element of a complete probability space $(\Omega, \Theta, \mathrm{P}), Z: \Omega \rightrightarrows \mathbb{R}^{n} \times \mathbb{R}^{m}$ is a point-to-set mapping representing the upper-level constraints, and $S: \mathbb{R}^{n} \times \Omega \rightarrow \mathbb{R}^{m}$ now defines the set of solutions to a lower-level parametric variational inequality problem of the form

$$
S(x, \omega):=\left\{y \in \mathbb{R}^{m} \mid-F(x, y, \omega) \in N_{C}(y)\right\},
$$

where

$$
F(x, y, \omega):=\left(\begin{array}{c}
\Lambda^{\mathrm{T}} t(x, v, \omega)-\Gamma \pi \\
\Gamma^{\mathrm{T}} h-g(x, \pi, \omega) \\
v-\Lambda h
\end{array}\right)
$$

In this hierarchical model we take into account possible uncertainties in the modelling of the travel costs and/or demands when evaluating the upper-level objective function for a given value of the parameters in $x$; further, we take the uncertainties into account in the optimization by minimizing the expectation of these objective values over the uncertainty set. In the case of the travel time/cost function, the random element $\omega$ can represent variations in road conditions as well as in properties regarding the trip-makers. Variations and uncertainties in the demand elasticities are also naturally reflected through the random element $\omega$ in the demand function. (See, e.g., Buchanan 1969 for an early discussion on the effect of market demand uncertainties on proper taxation schemes.)

Due to the fact that we study stochastic models of network control and pricing, we here effectively rule out from consideration such classic cases as that which models 'first-best' toll pricing, including, for example, the marginal cost pricing concept in the case of seeking a system optimal flow (e.g., Dafermos \& Sparrow 1969; Sender \& Netter 1970). This is a kind of inverse mathematical model, where a desired link flow is fixed and the only variables are the tolls to be set, typically on every link, in order to reproduce this flow as a traffic equilibrium one; see Larsson \& Patriksson $(1998,1999)$ and Larsson et al. (2003) for further discussions on this topic, and Marcotte \& Patriksson (2007) for a select bibliography on link toll pricing especially for obtaining system (or, social) optimal flows. Our model, in the context of link tolls, therefore belongs to the 'second-best' school, as discussed, for example, by Lawphongpanich \& Hearn (2004) and Yang et al. (2005).

We note that, in isolation, simple cases of the inclusion (10) have been studied previously. In, e.g., Gwinner \& Raciti (2006), the traffic model is a fixed demand one where data in both the demand and in the travel costs are stochastic, and the goal is to find the mean equilibrium solution as well as the variance in the equilibrium flows.

We next restate Theorem 1 in the context of this problem.

THEOREM 3 (existence of optimal solutions) Suppose that the following assumptions are fulfilled: the mappings $t(x, \cdot), g(x, \cdot)$ and $Z_{x}(\cdot)$ are measurable for every $x$; the set $Z(\omega)$ is closed for almost every $\omega \in \Omega$; the function $f$ is continuous in $(x, y)$, measurable in $\omega$, uniformly weakly coercive with respect to $x$ over the set $X$, and bounded from below by a $(\Theta, P)$-integrable function; for every $x \in X$, there is a neighborhood $U_{x} \ni x$ such that the set $\cup_{\bar{x} \in U_{x}} Z_{\bar{x}}(\omega)$ is bounded for almost every $\omega$; and the set $\Upsilon_{x_{0}}(\omega)$ is nonempty for some $x_{0} \in X$ and almost every $\omega$. Then, the problem $\left(\mathrm{SHTM}_{\Omega}\right)$ has at least one optimal solution.

Again, a corollary to follow shows that the absence of joint upper-level constraints makes life easier. 
Corollary 4 (existence of optimal solutions) Suppose that the upper-level feasible set $Z$ is independent of $y$, and hence the upper-level constraints can be written as $x \in X \subseteq \mathbb{R}^{n}$. Then, the above assumptions can be replaced by the following: the mappings $t(x, \cdot)$ and $g(x, \cdot)$ are measurable for every $x$; the set $X$ is closed; and the function $f$ is continuous in $(x, y)$, measurable in $\omega$, uniformly weakly coercive with respect to $x$ over the set $X$, and bounded from below by a $(\Theta, P)$-integrable function.

Notice that the most important technical condition to check here is that of the uniform weak coercivity of $f$, which is fulfilled automatically whenever $X$ is bounded. Since this is a quite natural assumption to make, any realistic application of the SMPEC to a traffic context is one where optimal designs exist.

\section{Solution stability}

The question of the continuity of optimal solutions to $\left(\mathrm{SMPEC}_{\Omega}\right)$ with respect to changes in the probability distribution is of great importance. From the computational point of view, a positive answer to this question would allow us to solve the problem by approximating the underlying probability measure with a sequence of discrete probability measures, and hence approximating the original infinite-dimensional optimization problem with a sequence of finite-dimensional optimization problems. From the theoretical point of view, we would conclude the robustness of the resulting optimal solutions with respect to errors in the modelling of uncertainty.

In structural optimization, owing to the anticipated fact that the real probability measure is unknown, and owing to the reported high sensitivity of the solutions with respect to small changes in the probability measure, many probability-free worst-case (pessimistic) models of uncertainty have been utilized as alternatives to probabilistic models, cf. Section 1. These approaches do yield stable structures, but do not take into account the probability of occurrence of the different scenarios, thereby often resulting in unnecessarily costly designs. In the area of structural optimization, there are example problems where the optimal solutions do not change continuously even if the probability distribution measure changes only locally (that is, only the density of the probability measure changes); cf. Evgrafov et al. (2003). There are example models in the area where no joint upper-level constraints are present, and whose optimal solutions are robust (Evgrafov et al. 2003), and there are also examples where a possible reformulation (that is, relaxation) of a non-robust problem yields a robust model (Evgrafov \& Patriksson 2003b).

Certain convex problems in stochastic programming have been proven robust (e.g., Robinson \& Wets 1987; Römisch \& Schultz 1991; Römisch 2003; Henrion \& Römisch 2004; and Heitsch et al. 2006), in some cases even with quantitative stability measures. Since stochastic programming is an instance of the framework of $\left(\mathrm{SMPEC}_{\Omega}\right)$ (e.g., Patriksson \& Wynter 1999, and Shapiro 2006), the latter clearly contains robust models. As remarked in Evgrafov \& Patriksson (2004), however, finding generic conditions on the mappings $Z$ and $S$ under which one can expect a continuous behaviour of the optimal solutions to non-convex models within the framework of $\left(\mathrm{SMPEC}_{\Omega}\right)$ (or at least the continuous behaviour of the optimal values of the upper-level variables $x$ ) remains an open problem.

We establish in this section that optimal solutions to $\left(\mathrm{SHTM}_{\Omega}\right)$ are stable under natural conditions when there are no joint upper-level constraints. In order to do so, we utilize a technique developed in previous work on structural topology optimization (Evgrafov \& Patriksson 2003b; and Evgrafov et al. 2003).

Consider a sequence $\left\{\mathrm{P}_{k}\right\}$ of probability measures defined on $\mathcal{B}(\Omega)$, together with the associated sequence of optimization problems:

$$
\left(\mathrm{SHTM}_{\Omega}\right)^{k}\left\{\begin{aligned}
\underset{(x, y(\cdot))}{\operatorname{minimize}} & \mathrm{E}_{\omega}[f(x, y(\omega), \omega)]:=\int_{\Omega} f(x, y(\omega), \omega) \mathrm{P}_{k}(d \omega), \\
\text { subject to } \quad x & \in X, \\
y(\omega) & \in S(x, \omega), \quad \mathrm{P}_{k} \text {-a.s. }
\end{aligned}\right.
$$

We assume that each measure $\mathrm{P}_{k}$ has a density $p_{k}(\cdot)$ with respect to a Lebesgue measure on $\Omega$ and that the sequence $\left\{p_{k}(\cdot)\right\}$ converges to a density $p(\cdot)$ of $\mathrm{P}$ Lebesgue-almost everywhere. The existence of densities is not a very restrictive assumption from the theoretical point of view. In what follows, the notation val $\mathrm{W}$ refers to the optimal objective value of the problem $\mathrm{W}$.

We then have the following result for the problem $\left(\mathrm{SMPEC}_{\Omega}\right)$.

THEOREM 5 (stability of optimal solutions) In addition to the conditions of Theorem 3 suppose that the mapping $t(x, \cdot, \omega)$ is strictly monotone for each $x \in X$ and $\omega \in \Omega$ and that $g$ is either a constant mapping 
in $\pi$ or $-g(x, \cdot, \omega)$ is strictly monotone for each $x \in X$ and $\omega \in \Omega$. Suppose that the sequence $\left\{\mathrm{P}_{k}\right\}$ of probability measures weakly converges to $\mathrm{P}$ and that $\left(x_{k}, y_{k}(\cdot)\right)$ solves $\left(\mathrm{SHTM}_{\Omega}\right)^{k}$ for each $k$. Then, each limit point (and there is at least one) of the sequence $\left\{\left(x_{k}, y_{k}(\cdot)\right)\right\}$ is an optimal solution to $\left(\mathrm{SHTM}_{\Omega}\right)$.

Proof. Consider an optimal solution $\left(x^{*}, y^{*}(\cdot)\right)$ to $\left(\mathrm{SMPEC}_{\Omega}\right)$. Thanks to the properties of $t(x, \cdot, \omega)$ and $g(x, \cdot, \omega)$ the components $v$ and $\pi$ of the mapping $y$ are continuous in $x$ and $\omega$ and the $h$ component therefore also belongs to a bounded set (e.g., Marcotte \& Patriksson, 2007). It follows, since $f$ is infcompact, that any sequence of feasible designs and responses is bounded and hence has a limit point. The optimal solution $\left(x^{*}, y^{*}(\cdot)\right)$ to $\left(\mathrm{SMPEC}_{\Omega}\right)$ is moreover feasible in $\left(\mathrm{SMPEC}_{\Omega}\right)^{k}$ for all $k$. It follows that $\operatorname{val}\left(\mathrm{SHTM}_{\Omega}\right) \geq \lim \sup _{k \rightarrow \infty} \operatorname{val}\left(\mathrm{SHTM}_{\Omega}\right)^{k}$.

Conversely, let $\left\{\left(x_{k}, y_{k}(\cdot)\right)\right\}$ be a sequence of optimal solutions to $\left(\mathrm{SMPEC}_{\Omega}\right)^{k}$. By inf-compactness, this sequence is bounded; let $(\bar{x}, \bar{y}(\cdot))$ denote a limit point, which moreover automatically is feasible for almost every $\omega$ in $\left(\mathrm{SMPEC}_{\Omega}\right)$. Then, also utilizing the lower semi-continuity of $f$ and Fatou's Lemma, we obtain that

$$
\begin{aligned}
\operatorname{val}\left(\operatorname{SHTM}_{\Omega}\right) & \leq \int_{\Omega} f(\bar{x}, \bar{y}(\omega), \omega) p(\omega) d \omega \\
& \leq \int_{\Omega} \liminf _{k \rightarrow \infty} f\left(x_{k}, y_{k}(\omega), \omega\right) p(\omega) d \omega \\
& \leq \liminf _{k \rightarrow \infty} \int_{\Omega} f\left(x_{k}, y_{k}(\omega), \omega\right) p_{k}(\omega) d \omega \\
& =\liminf _{k \rightarrow \infty} \operatorname{val}\left(\operatorname{SHTM}_{\Omega}\right)^{k}
\end{aligned}
$$

The combination of the two inequalities thus derived implies the result sought.

The conditions of the theorem are naturally satisfied in applications. First, the strict monotonicity assumption on the mappings describing the link travel cost and the negative of the demand function is standard in the literature on traffic equilibria. Further, any continuous probability distribution used in practice, such as the uniform and normal distributions, has a density. This means, for example, that according to the above theorem, small changes in, say, the support of a uniform distribution, leads to small changes in the optimal design. The distribution of discrete random variables however does not have a density. The above theorem can therefore not be utilized to assert that discretization approaches converge. This is, however, the topic of Section 5.2.

Establishing the stability of globally optimal solutions to special SMPEC models with joint upperlevel constraints is an interesting avenue for future research. Similarly, the stability of stationary points or locally optimal design solutions certainly are of interest to obtain, as they are the likely vectors that can be reached with most numerical methods. See also the future research discussions in Section 6 .

\section{Solution approaches}

\subsection{Inexact penalization}

Compared to one-level problems, bilevel optimization algorithms are much less straightforward to develop owing to the non-convex nature of the problem and the absence of constraint qualifications for nonlinear programming (Luo et al. 1996; Scheel \& Scholtes 2000). One approach is to move the equilibrium constraint as a penalty into the objective function. For examples of penalty functions leading to algorithmic constructions to MPEC, see Luo et al. (1996), Pang (1997), Ye et al. (1997), Scholtes \& Stöhr (1999), and $\mathrm{Hu} \& \mathrm{Ralph}$ (2004). In particular, exact penalties are of great importance, since they lead to exact solutions while they do not require the penalty parameter to tend to infinity (Burke 1991). However, one cannot expect to be able to construct an exact penalty for SMPEC problems, given an exact penalty for each $\omega$, as an example in Evgrafov \& Patriksson (2004) illustrates. Again, the reason is the presence of the coupling upper-level constraints. The following approach is however possible; the result is quoted from Evgrafov \& Patriksson (2004, Theorem 4.1), and is a rather straightforward type of result for penalty methods in nonlinear programming.

THEOREM 6 (inexact penalization of lower-level constraints) Suppose the conditions of Theorem 1 are satisfied, so that there exists an optimal solution to $\left(\operatorname{SMPEC}_{\Omega}\right)$. Let also the function $(x, y, \omega) \mapsto$ 
$G(x, y, \omega)$ be non-negative, continuous in $(x, y)$ for almost every $\omega$, measurable in $\omega$ for every $(x, y) \in$ $\mathbb{R}^{n} \times \mathbb{R}^{m}$, and such that

$$
S(x, \omega)=\left\{y \in \mathbb{R}^{m} \mid G(x, y, \omega)=0\right\} .
$$

Then, the penalized problem

$$
\left(\operatorname{SMPEC}_{\Omega}\right)_{\mu}^{1}\left\{\begin{array}{l}
\underset{(x, y(\cdot))}{\operatorname{minimize}} \mathrm{E}_{\omega}[f(x, y(\omega), \omega)+\mu G(x, y, \omega)], \\
\text { subject to }(x, y(\omega)) \in Z(\omega), \quad \text { P-a.s., }
\end{array}\right.
$$

has an optimal solution for every $\mu \geq 0$ and

$$
\sup _{\mu \geq 0} \operatorname{val}\left(\mathrm{SMPEC}_{\Omega}\right)_{\mu}^{1}=\lim _{\mu \rightarrow \infty} \operatorname{val}\left(\mathrm{SMPEC}_{\Omega}\right)_{\mu}^{1}=\operatorname{val}\left(\mathrm{SMPEC}_{\Omega}\right) .
$$

Furthermore, any limit point of the sequence of upper-level optimal solutions $\left\{x_{\mu}\right\}$ to $\left(\mathrm{SMPEC}_{\Omega}\right)_{\mu}^{1}$ (and there is at least one) is an upper-level optimal solution to $\left(\mathrm{SMPEC}_{\Omega}\right)$.

While we in the above approach lift the equilibrium constraints into the objective, thus reducing the original bilevel optimization problem into a series of one-level problems, we have previously argued that joint upper-level constraints imply complications and may therefore alternatively conclude that it is the constraints that ' $(x, y(\omega)) \in Z(\omega)$ holds P-almost surely' that should be penalized in order to turn the problem into a more 'standard' SMPEC model. (This is indeed an approach that has been utilized previously; see, e.g., Evgrafov \& Patriksson 2003a.) Providing a general theory of such an approach is a quite interesting avenue for future research. For now, we establish the correctness of such an approach in the transportation domain.

Theorem 7 (inexact penalization of upper-level constraints) Suppose the conditions of Theorem 5 are satisfied. Let also the function $(x, y, \omega) \mapsto G(x, y, \omega)$ be non-negative, continuous in $(x, y)$ for almost every $\omega$, measurable in $\omega$ for every $(x, y) \in \mathbb{R}^{n} \times \mathbb{R}^{m}$, and such that

$$
Z(\omega)=\left\{(x, y) \in \mathbb{R}^{n} \times \mathbb{R}^{m} \mid G(x, y, \omega)=0\right\} .
$$

Then, the penalized problem

$$
\left(\operatorname{SHTM}_{\Omega}\right)_{\mu}^{2}\left\{\begin{array}{l}
\underset{(x, y(\cdot))}{\operatorname{minimize}} \mathrm{E}_{\omega}[f(x, y(\omega), \omega)+\mu G(x, y, \omega)], \\
\text { subject to } y(\omega) \in S(x, \omega), \quad \text { P-a.s. }
\end{array}\right.
$$

has an optimal solution for every $\mu \geq 0$ and

$$
\sup _{\mu \geq 0} \operatorname{val}\left(\operatorname{SHTM}_{\Omega}\right)_{\mu}^{2}=\lim _{\mu \rightarrow \infty} \operatorname{val}\left(\operatorname{SHTM}_{\Omega}\right)_{\mu}^{2}=\operatorname{val}\left(\operatorname{SHTM}_{\Omega}\right) .
$$

Furthermore, any limit point of the sequence of upper-level optimal solutions $\left\{x_{\mu}\right\}$ to $\left(\mathrm{SHTM}_{\Omega}\right)_{\mu}^{2}$ (and there is at least one) is an upper-level optimal solution to $\left(\mathrm{SHTM}_{\Omega}\right)$.

Proof. For every $\mu \geq 0$ the problem $\left(\mathrm{SHTM}_{\Omega}\right)_{\mu}^{2}$ satisfies the assumptions of Theorem 3 . Let us denote by $\left(x_{\mu}, y_{\mu}(\cdot)\right)$ an arbitrary optimal solution to $\left(\mathrm{SHTM}_{\Omega}\right)_{\mu}^{2}$. Following the proof of Lemma 9.2.1 and Theorem 9.2.2 in Bazaraa et al. (1993) we get

$$
\operatorname{val}\left(\operatorname{SHTM}_{\Omega}\right) \geq \sup _{\mu \geq 0} \operatorname{val}\left(\mathrm{SHTM}_{\Omega}\right)_{\mu}^{2}=\lim _{\mu \rightarrow \infty} \operatorname{val}\left(\mathrm{SHTM}_{\Omega}\right)_{\mu}^{2}=\lim _{k \rightarrow \infty} \mathrm{E}_{\omega}\left[f\left(x_{\mu_{k}}, y_{\mu_{k}}(\omega), \omega\right)\right],
$$

for some sequence $\left\{\mu_{k}\right\}$ with $\mu_{k} \rightarrow \infty$.

By the uniform coercivity (an assumption of Theorem 3 ) of $f$ in $x$, and by the properties of a penalty function $G$, the sequence $\left\{x_{\mu_{k}}\right\}$ is bounded. Switching to a subsequence if necessary, we may assume that $\lim _{k \rightarrow \infty} x_{\mu_{k}}=\tilde{x}$.

Owing to the lower boundedness of $f$ (an assumption of Theorem 3), we have that

$$
\lim _{k \rightarrow \infty} \mathrm{E}_{\omega}\left[f\left(x_{\mu_{k}}, y_{\mu_{k}}(\omega), \omega\right)\right] \geq \mathrm{E}_{\omega}\left[\liminf _{k \rightarrow \infty} f\left(x_{\mu_{k}}, y_{\mu_{k}}(\omega), \omega\right)\right] .
$$


By using the same boundedness arguments for the sequence $\left\{y_{\mu_{k}}(\omega)\right\}$ of responses as in the proof of Theorem 5, we can conclude that there is a sequence $k(\omega)$ such that $y_{\mu_{k(\omega)}} \rightarrow \bar{y}(\omega)$ and

$$
\liminf _{k \rightarrow \infty} f\left(x_{\mu_{k}}, y_{\mu_{k}}(\omega), \omega\right)=\lim _{k(\omega) \rightarrow \infty} f\left(x_{\mu_{k(\omega)}}, y_{\mu_{k(\omega)}}(\omega), \omega\right) \geq f(\tilde{x}, \bar{y}(\omega), \omega),
$$

for P-almost every $\omega$. Owing to the closedness of $S$ (due to the continuity of $t$ and $g$ and the strict monotonicity of $t(x, \cdot)$ and $-g(x, \cdot)$ for every $x)$, it follows that $\bar{y}(\omega) \in S(\tilde{x}, \omega)$, P-almost surely. Following the proof of Theorem 9.2.2 of Bazaraa et al. (1993) we get

$$
0=\lim _{k \rightarrow \infty} \mathrm{E}_{\omega}\left[G\left(x_{\mu_{k}}, y_{\mu_{k}}(\omega), \omega\right)\right] \geq \mathrm{E}_{\omega}\left[\liminf _{k \rightarrow \infty} G\left(x_{\mu_{k}}, y_{\mu_{k}}(\omega), \omega\right)\right]
$$

and

$$
\liminf _{k \rightarrow \infty} G\left(x_{\mu_{k}}, y_{\mu_{k}}(\omega), \omega\right) \geq G(\tilde{x}, \bar{y}(\omega), \omega) \geq 0,
$$

for P-almost every $\omega$ by the continuity and non-negativity of $G$, thus showing that $(\tilde{x}, \bar{y}(\omega)) \in Z(\omega)$ for P-almost every $\omega$.

Considering the parametric optimization problem in the variables $y(\omega)$ to

$$
\begin{array}{rr}
\operatorname{minimize} & f(\tilde{x}, y(\omega), \omega), \\
\text { subject to }(\tilde{x}, y(\omega)) & \in Z(\omega), \quad \text { P-a.s., } \\
y(\omega) & \in S(\tilde{x}, \omega), \quad \text { P-a.s., }
\end{array}
$$

we can apply Theorem 8.2.11 in Aubin \& Frankowska (1990) to obtain the existence of a measurable function $\tilde{y}(\omega)$ such that $f(\tilde{x}, \tilde{y}(\omega), \omega) \leq f(\tilde{x}, \bar{y}(\omega), \omega)$ P-almost surely, thus showing that

$$
\sup _{\mu \geq 0} \operatorname{val}\left(\mathrm{SHTM}_{\Omega}\right)_{\mu}^{2} \geq \mathrm{E}_{\omega}[f(\tilde{x}, \tilde{y}(\omega), \omega)] \geq \operatorname{val}\left(\mathrm{SHTM}_{\Omega}\right) .
$$

Together with (12), this proves the claim.

\subsection{Discretization approaches}

A popular method for solving a stochastic programming problem involving a non-discrete probability measure is to approximate it with a sequence of finite-dimensional problems with discrete measures. To implement such a procedure one needs a discrete measure $\tilde{P}$, in some sense close to the original one. A common choice is to start from a finite partition $\mathcal{A}=\left\{\Omega_{i} \mid \cup_{i} \Omega_{i}=\Omega\right\}$ and define its probability as $\tilde{P}_{i}=P\left(\Omega_{i}\right)$. This involves the calculation of probabilities which we may want to avoid and replace by estimations. We also need approximations $\tilde{q}$ of random elements $q$ of the original problem, which could involve conditional means. Instead we would like to choose sampling points $\tilde{\omega}_{i} \in \Omega_{i}$ and set, as before, $\tilde{q}_{i}=q\left(\tilde{\omega}_{i}\right)$.

Examples of discretizations of stochastic programming problems with recourse based on the ideas of conditional means can be found in Olsen (1976), Birge \& Wets (1986). One generic scheme that allows us to avoid such computations is the 'method of mechanical quadratures' (Vainikko 1971) which will be used below; it was successfully used to discretize stochastic programming problems with complete recourse in Lepp (1990), and for stochastic structural topology optimization problems in Evgrafov and Patriksson $(2003 \mathrm{a}, \mathrm{b})$.

Suppose $\Omega$ is a compact metric space with a metric $\rho(\cdot, \cdot)$. Let $\Theta \supset \mathcal{B}(\Omega), \mathrm{P}\left(\left\{\omega \mid \rho\left(\omega, \omega_{0}\right)<r\right\}\right)=$ $\mathrm{P}\left(\left\{\omega \mid \rho\left(\omega, \omega_{0}\right) \leq r\right\}\right)$ for every $\omega_{0} \in \Omega, r>0$, and $\mathrm{P}$ is a regular measure. Consider a sequence of partitions of $\Omega, \mathcal{A}^{k}=\left\{A_{1}^{k}, \ldots, A_{k}^{k}\right\}$, satisfying the following properties for each $k$ and $1 \leq l \leq k$ : $\mathrm{P}\left(A_{l}^{k}\right)>0 ; \cup_{l=1}^{k} A_{l}^{k}=\Omega ; A_{i}^{k} \cap A_{j}^{k}=\emptyset, i \neq j ; \lim _{k \rightarrow \infty} \operatorname{diam}\left(A_{l}^{k}\right)=0 ;$ and $\mathrm{P}\left(\partial A_{l}^{k}\right)=0$. The collection of sets $\mathcal{A}^{k}$ generates an algebra $\Theta_{0} \subset \Theta$.

Define a sequence of discrete measures $\mathrm{P}_{k}$ with support $\operatorname{supp} \mathrm{P}_{k}=\left\{\omega_{1}^{k}, \ldots, \omega_{k}^{k}\right\}$, satisfying the following properties for each $k$ and $1 \leq l \leq k: \omega_{l}^{k} \in A_{l}^{k}$; and $\lim _{k \rightarrow \infty} \max _{1 \leq l \leq k} \mathrm{P}_{k}\left(\omega_{l}^{k}\right) / \mathrm{P}\left(A_{l}^{k}\right)=1$.

We further assume that the functions $t(x, \cdot), g(x, \cdot)$ and $f(x, \cdot)$ are $\Theta_{0}$-measurable and bounded. The problem (10)-(11) transforms into the following:

$$
S\left(x, \omega_{l}^{k}\right):=\left\{y_{l}^{k} \in \mathbb{R}^{m} \mid-F\left(x, y_{l}^{k}, \omega_{l}^{k}\right) \in N_{C}\left(y_{l}^{k}\right)\right\},
$$


with

$$
y_{l}^{k}:=\left(\begin{array}{c}
h_{l}^{k} \\
\pi_{l}^{k} \\
v_{l}^{k}
\end{array}\right) \quad \text { and } \quad F\left(x, y_{l}^{k}, \omega_{l}^{k}\right):=\left(\begin{array}{c}
\Lambda^{\mathrm{T}} t\left(x, v_{l}^{k}, \omega_{l}^{k}\right)-\Gamma \pi_{l}^{k} \\
\Gamma^{\mathrm{T}} h_{l}^{k}-g\left(x, \pi_{l}^{k}, \omega_{l}^{k}\right) \\
v_{l}^{k}-\Lambda h_{l}^{k}
\end{array}\right) .
$$

The discretized collection of problems has the following appearance:

$$
(\mathrm{SHTM})^{k}\left\{\begin{array}{l}
\underset{\left(x, y_{1}^{k}, \ldots, y_{k}^{k}\right)}{\operatorname{minimize}} \sum_{l=1}^{k} P_{k}\left(\omega_{l}^{k}\right) f\left(x, y_{l}^{k}\left(\omega_{l}^{k}\right),\right. \\
\text { subject to } \quad x \in X, \\
y_{l}^{k}\left(\omega_{l}^{k}\right) \in S\left(x, \omega_{l}^{k}\right), \quad l=1, \ldots, k .
\end{array}\right.
$$

Let $\left(\left(x^{k}\right)^{*},\left(y^{k}\right)^{*}\right)$ denote an optimal solution to the problem (SHTM $)^{k}$, where the vector $y^{k}$ is the concatenation of the vectors $y_{l}^{k}, l=1, \ldots, k$. The following result can be obtained.

THEOREM 8 (convergence of discretized solutions) In addition to the conditions of Theorem 3 suppose that the mapping $t(x, \cdot, \omega)$ is strongly monotone for each $x \in X$ and $\omega \in \Omega$ and that $g$ is either a constant mapping in $\pi$ or $-g(x, \cdot, \omega)$ is strongly monotone for each $x \in X$ and $\omega \in \Omega$. Consider the sequence $\left\{(\mathrm{SHTM})^{k}\right\}$ of discretizations of the problem $\left(\mathrm{SHTM}_{\Omega}\right)$. Then, any limit point of the sequence $\left\{\left(\left(x^{k}\right)^{*},\left(y^{k}\right)^{*}\right)\right\}$ of optimal solutions to these problems (and there is at least one such point) is an optimal solution to the problem $\left(\mathrm{SHTM}_{\Omega}\right)$.

Proof. We first remark that it is not difficult to establish that every limit state vector $\bar{y}$ of the sequence $\left\{\left(y^{k}\right)^{*}\right\}$ is not only feasible in the limit lower level problem corresponding to a limit $\bar{x}$ of the sequence $\left\{\left(x^{k}\right)^{*}\right\}$ but it also solves this limit problem; this is due to the boundedness of these sequences and the integrability of the lower level solution (cf. Castaing \& Valadier 1977, Lemma III.39; and Lepp 1994, Remark 2). Moreover the discrete convergence of the $(v, \pi)$-components is strong. The remainder of the proof is similar to that of Theorem 5; see also Evgrafov \& Patriksson (2003a).

Notice that the strong monotonicity assumption is introduced in the above result in order to be able to infer the strong regularity of the equilibrium solution, which in fact can be established under slightly less stringent assumptions (e.g., Patriksson \& Rockafellar 2003, and Patriksson 2004).

The above algorithm is similar to a Monte Carlo technique used previously in stochastic programming and for some special cases of SMPEC models. It is known as the sample path method, sample average approximation, stochastic counterpart method, and the simulated likelihood method (see, e.g., Plambeck et al. 1993, 1996; Rubinstein \& Shapiro 1993; Robinson 1996; Gürkan et al. 1998, 1999a,b; Kleywegt et al. 2001; Shapiro 2003, 2006, 2008; Shapiro \& Xu 2005; Meng \& Xu 2006; and the references therein). Using $N$ random samples $\omega^{1}, \omega^{2}, \ldots, \omega^{N}$ of the random vector $\omega$, a problem of the form

$$
(\mathrm{SHTM})^{N}\left\{\begin{aligned}
\underset{\left(x, y_{k}\right)}{\operatorname{minimize}} & \frac{1}{N} \sum_{k=1}^{N} f\left(x, y^{k}\right), \\
\text { subject to } \quad x \in X, & \\
y^{k} & \in S\left(x, \omega^{k}\right), \quad k=1, \ldots, N
\end{aligned}\right.
$$

is solved, for increased values of $N$.

Its main difference to the discretization approach resulting in the problem (SHTM) $)^{k}$ above is that the sampling is made such that the scenario probabilities all are the same. Shapiro \& Xu (2005) study the convergence of this method for $(\mathrm{SMPCC})_{\Omega}$ under conditions that, translated into our notation, are fulfilled if $y(\cdot, \omega)$ is unique and continuous for almost every $\omega, X$ is non-empty and compact, and the function $f(x, y(x, \cdot), \cdot)$ is bounded over $X$ almost surely. Under additional conditions on $S$ and $f$ such that the expected value function is differentiable, it is also established in Shapiro \& Xu (2005) that stationary points to discretized versions of $(\mathrm{SMPCC})_{\Omega}$ converge to the set of stationary points to (SMPCC) $\Omega$. Because of the smoothing effect of the averaging operation, it is remarked that differentiability of the expected value function may hold in many more cases than for the non-deterministic problem (MPCC). We expect the same to hold for $(\mathrm{SHTM})_{\Omega}$. 
The conditions imposed in the similar work in Gürkan et al. (1999a) in particular imply that the lower-level problem has strongly regular solutions (Robinson 1980), but on the other hand the result quite immediately extends to inexact solutions of the approximate problems $(\mathrm{SHTM})^{N}$. (Notice also that the conditions of the above theorem imply the strong regularity of the lower-level solution in the relevant entities, cf. Yen 1995; Patriksson \& Rockafellar 2003; and Patriksson 2004.)

In Birbil et al. (2006) the above-mentioned sample path method was applied to a special case of $(\mathrm{SMPCC})_{\Omega}$ modelling a toll pricing problem. Using the classic separable polynomial BPR travel cost functions (US Bureau of Public Roads 1964) in a fixed demand traffic assignment model, the problem is stated as follows: Suppose that tolls are to be set during the beginning of a fairly long time period, during which time the tolls cannot be altered. The parameters of the BPR formula are considered stochastic in order to represent varying road conditions during this time period, and the model $(\mathrm{SMPCC})_{\Omega}$ then represents a means to derive the best link tolls 'on average'. A test case using the Sioux Falls network (LeBlanc et al. 1975), where $n=|\mathcal{L}|=76$, is reported; in this example the exact solution can be computed before-hand, making it possible to establish, in this case, convergence to an optimal solution.

The main interest in the above techniques is of course that SMPEC models can be solved through a sequence of MPEC models, for which relatively efficient algorithms exist (e.g., Fletcher et al. 2006; Ralph 2008; and references therein). Shapiro (2006) establishes that convergence in some cases is exponential, which implies that relatively few samples may be needed to reach at least a near-optimal (or, nearstationary) solution; numerical experiments in Shapiro \& Xu (2005) and Birbil et al. (2006) confirm this belief at least in small-scale examples.

Therefore, there is a hope that SMPEC traffic models can be solved approximately with a computational effort that is not several orders of magnitude larger than for one instance of a discretized problem. In particular, a rough design solution that is at least a lot better than any individual MPEC solution can probably be achieved through the solution of one single problem of the form (SHTM) ${ }^{k}$ or $(\text { SHTM })^{N}$, provided of course that the samples are well chosen. The analysis performed in this paper in fact - to at least some degree - validates such a simple approach, through the combined results of Theorems 5 and 8.

\section{$6 \quad$ Final remarks and future research directions}

While the lower-level traffic model defined in Section 3 is built upon a deterministic (possibly multi-class) traffic equilibrium model with inelastic or elastic demands, the above development can be performed based also upon a stochastic model. Indeed, the properties needed for our theoretical results are not connected to the route-choice principles per se, but rather to the properties of the equilibrium solution. The main property needed is that the lower-level optimal link flow and demand is closed (in some cases continuous) as a function of $x$ and $\omega$, which is a property present also for, say, the logit-based stochastic model of Fisk (1980) (see also Patriksson 2004, for a sensitivity analysis of this model) and for probit models (e.g., Von Falkenhausen 1966; Daganzo \& Sheffi 1977; Daganzo 1983; Davis 1994; and Maher et al. 2001; see also the sensitivity analyses in Clark \& Watling $(2000,2002,2005)$. For a recent account of research done on link tolling under stochastic user equilibrium, see Stewart (2007). As far as this author is aware, the present paper is the first that (essentially) covers an analysis of a stochastic MPEC model where the lower level model is a stochastic user equilibrium (SUE) model; a standard MPEC model built on a lower-level SUE traffic model does not constitute an SMPEC model.

As mentioned already in Section 1.3, congestion control in communication networks is a very important topic; its relationships to traffic MPEC models were also alluded to. Several parameters in the definition of the problem through whose solution one determines the transmission rates are likely to be subject to random fluctuations and are also difficult to measure exactly, in particular the capacity of the links (in terms of flow rates). Therefore, it seems natural to study stochastic models of transmission control, which then would have the form of SMPEC problems.

A natural future research task is to investigate the possible convergence of inexact solutions to the subproblems in the algorithms investigated in this paper to (in)exact solutions to the original one. Along these lines, it would be interesting also to analyze and extend to our situation a convergence result in Gürkan et al. (1999a): In their application of the sample path method the maximum value of $N$ (that is, the total number of samples) required to reach a given accuracy of the final solution while also solving each subproblem inexactly grows linearly with the number $n$ of design parameters.

An interesting research issue related to the above is whether stability results extending that for globally optimal solutions can be verified also for locally optimal design solutions, and/or solutions to a suitably 
stated necessary (or, stationarity) condition for a local optimum. The latter conditions are certainly interesting in their own light, as KKT-type optimality conditions for general SMPEC problems are not well developed yet. Results along these lines are currently under investigation.

Another interesting question is whether convergence is achieved for the natural combination of penalization and discretization; it has previously been successfully applied and established in structural topology optimization in Evgrafov \& Patriksson (2003a).

A related issue regards the stability of optimal solutions to SMPEC traffic models with joint upperlevel constraints. We have in Section 4 described the unlikelihood of the existence of such a result for SMPEC traffic models in general, but we have also related to work in Evgrafov \& Patriksson (2003b) that has showed that certain relaxations of joint upper-level constraints (in particular stress constraints in topology optimization problems) may in fact induce robustness. The form of such relaxations is naturally highly dependent on the type of constraints considered. It would be interesting to investigate the existence of good relaxations for particular classes of joint upper-level constraints in the SMPEC traffic framework that would induce robustness. It would also be of interest to learn whether any sound solution approaches could be derived from them. Indeed, in topology optimization such algorithms are now quite well-known and established; see, e.g., Kirsch (1990), Rozvany \& Birker (1994), Cheng \& Guo (1997), Guo et al. (2001), Petersson (2001), Stolpe \& Svanberg (2001), and Evgrafov \& Patriksson (2003b, 2005) for both classic work on the 'stress singularity phenomenon' and specially designed relaxation methods for dealing with stress constraints. In relation to the negative result in Evgrafov \& Patriksson (2003b) that one cannot expect convergence of a scheme in which $\left\{\mathrm{P}_{k}\right\}$ converges to $\mathrm{P}$ and the relaxation parameter tends to zero simultaneously, one must observe that in that particular application the objective function of the lower-level variational inequality is not everywhere finite; the situation in the SMPEC traffic context should be more favourable.

A potentially interesting modification transforms the constraint that $\mathrm{P}_{\Omega}[(x, y(\omega)) \in Z(\omega)]=1$ holds into the relaxation that, for some given $\alpha \in(0,1), \mathrm{P}_{\Omega}[(x, y(\omega)) \in Z(\omega)] \geq \alpha$ holds. This is the classic 'probabilistic' or 'chance constraint' of stochastic programming (see Charnes et al. 1958; and Prékopa 2003) and the classic 'reliability constraint' from structural optimization. Whether interesting stability properties can be derived from an SMPEC model equipped with this relaxed joint upper-level constraint is an interesting future research question.

Applications to toll pricing and design problems in traffic networks have been mentioned previously. SMPEC models can of course also be utilized to similarly model and analyze networks of other commodities. One example is optical data communication networks where, for example, revenue maximization models (known as stochastic traffic engineering) involving bandwidth provisioning in the face of stochastic elastic bandwidth demands are discussed in Mitra \& Wang (2005). The analysis and numerical solution of this and other problems in communication networks within the framework of SMPEC, is a fruitful research avenue.

Finally, it would be interesting to investigate whether, in the context of network design and/or pricing the analysis of this paper may offer contributions to the study of reliability of traffic flows (see, e.g., Bell \& Iida 1997; Bell 2000; and Clark \& Watling 2005).

\section{Acknowledgments}

The research leading to this paper was performed partially in association with the Gothenburg Mathematical Modelling Centre (GMMC) at Mathematical Sciences (Chalmers University of Technology and Göteborg University), whose main sponsor is the Swedish Foundation for Strategic Research (SSF). A total of seven referees have provided remarks on the forerunner, the discussion paper (Patriksson 2008), and on previous versions of this paper; the presentation has benefited substantially from their contributions.

\section{References}

Aashtiani, H. Z. \& Magnanti, T. L. 1981 Equilibria on a congested transportation network. SIAM Journal on Algebraic and Discrete Methods 2, no. 3, pp. 213-226.

Abdulaal, M. \& LeBlanc, L. J. 1979 Continuous equilibrium network design models. Transportation Research Part B 13, no. 1, pp. 19-32.

Akçelik, R. 1991 Travel time functions for transport planning purposes: Davidson's function, its timedependent form and an alternative travel time function. Australian Road Research, 21), no. 1, pp. 44-59. 
Aubin, J.-P. \& Frankowska, H. 1990 Set-Valued Analysis. Boston, MA, USA: Birkhäuser Boston, Inc.

Auchmuty, G. 1989 Variational principles for variational inequalities. Numerical Functional Analysis and Optimization 10, no. 9-10, pp. 863-874.

Bard, J. F. 1998 Practical Bilevel optimization: Algorithms and Applications. Dordrecht, The Netherlands: Kluwer Academic Publishers.

Bazaraa, M. S., Sherali, H. D. \& Shetty, C. M. 1993 Nonlinear Programming: Theory and Algorithms, 2nd edn. New York, NY, USA: John Wiley \& Sons.

Beckmann, M. J., McGuire, C. B. \& Winsten, C. B. 1956 Studies in the Economics of Transportation. New Haven, CT, USA: Yale University Press.

Bell, M. G. H. 2000 A game theory approach to measuring the performance reliability of transport networks. Transportation Research Part B 34, no. 6, pp. 533-545.

Bell, M. G. H.\& Iida, Y. 1997. Transportation Network Analysis. Chichester, U.K.: John Wiley \& Sons.

Ben-Haim, Y. \& Elishakoff, I. 1990. Convex Models of Uncertainty in Applied Mechanics. Studies in Applied Mechanics. Amsterdam: Elsevier.

Ben-Tal, A., Boyd, S. \& Nemirovski, A. 2006 Extending scope of robust optimization: comprehensive robust counterparts of uncertain problems. Mathematical Programming 107, no. 1-2, pp. 63-89.

Ben-Tal, A., Kočvara, M., Nemirovski, A. \& Zowe, J. 1999 Free material design via semidefinite programming: the multiload case with contact conditions. SIAM Journal on Optimization 9, no. 4, pp. 813-832.

Ben-Tal, A. \& Nemirovski, A. 1997 Robust truss topology design via semidefinite programming. SIAM Journal on Optimization, 7, no. 4, pp. 991-1016.

Ben-Tal, A. \& Nemirovski, A. 1999 Robust solutions of uncertain linear programs. Operations Research Letters, 25, no. 1, pp. 1-13.

Ben-Tal, A. \& Nemirovski, A. 2002 Robust optimization-methodology and applications. Mathematical Programming, 92, no. 3, pp. 453-480.

Bendsøe, M. P. \& Sigmund, O. 2003 Topology Optimization: Theory, Methods, and Applications. Berlin: Springer-Verlag.

Birbil, S. I., Gürkan, G. \& Listes, O. 2006 Solving stochastic mathematical programs with complementarity constraints using simulation. Mathematics of Operations Research 31, no. 4, pp. 739-760.

Birge, J. R. \& Louveaux, F. 1997 Introduction to Stochastic Programming. Springer Series in Operations Research. New York, NY, USA: Springer-Verlag.

Birge, J. R. \& Wets, R. J.-B. 1986 Designing approximation schemes for stochastic optimization problems, in particular for stochastic programs with recourse, Mathematical Programming Study 27, 54-102.

Buchanan, J. M. 1969 External diseconomies, corrective taxes, and market structure. American Economic Review 59, no. 1, pp. 174-77.

Burke, J. V. 1991 An exact penalization viewpoint of constrained optimization. SIAM Journal on Control and Optimization 29, no. 4, pp. 968-998.

Cascetta, E. \& Nguyen, S. 1988 A unified framework for estimating or updating origin/destination matrices from traffic counts. Transportation Research Part B 22, no. 6, pp. 437-455.

Castaing, C. \& Valadier, M. 1977 Convex Analysis and Measurable Multifunctions. Lecture Notes in Mathematics, vol. 580. Berlin: Springer-Verlag.

Charnes, A., Cooper, W. W. \& Symonds, G. H. 1958 Cost horizons and certainty equivalents: An approach to stochastic programming of heating oil. Management Science 4, no. 3, pp. 235-263.

Chen, A., Subprasom, K. \& Ji, E. Z. 2006 A simulation-based multi-objective genetic algorithm (SMOGA) procedure for BOT network design problem. Optimization and Engineering 7, no. 3, pp. 225-247.

Chen, A., Yang, H., Lo, H. K. \& Tang, W. H. 2002 Capacity reliability of a road network: An assessment methodology and numerical results. Transportation Research Part B 36, no. 3, pp. 225-252.

Chen, Y. \& Florian, M. 1998 Congested O-D demand adjustment problem: Formulation and optimality 
conditions. In Multilevel Optimization: Algorithms and Applications (eds. A. Migdalas, P. Pardalos, \& P. Värbrand), pp. 1-20, vol. 20 in Nonconvex Optimization and Its Applications, Dordrecht, The Netherlands: Kluwer Academic Publishers.

Cheng, G. \& Guo, X. 1997 E-relaxed approach in structural topology optimization. Structural Optimization 13, no. 4, pp. 258-266.

Chiou, S. W. 2005 Bilevel programming for the continuous transport network design problem. Transportation Research Part B 39, no. 4, pp. 361-383.

Christiansen, S., Patriksson, M. \& Wynter, L. 2001 Stochastic bilevel programming in structural optimization. Structural and Multidisciplinary Optimization 21, no. 5, pp. 361-371.

Clark, S. D. \& Watling, D. P. 2000 Probit-based sensitivity analysis for general traffic networks, Transportation Research Record 1733, pp. 88-95.

Clark, S. D. \& Watling, D. P. 2002 Sensitivity analysis of the probit-based stochastic user equilibrium assignment model. Transportation Research Part B 36, no. 7, pp. 617-635 \& 851.

Clark, S. \& Watling, D. 2005 Modelling network travel time reliability under stochastic demand, Transportation Research Part B 39, no. 2, pp. 119-140.

Connors, R. D., Sumalee, A., \& Watling, D. P. 2007 Sensitivity analysis of the variable demand probit stochastic user equilibrium with multiple user-classes. Transportation Research Part B 41, no. 6, pp. 593615 .

Cree, N. D., Maher, M. J. \& Paechter, B. 1998 The continuous equilibrium optimal network design problem: A genetic approach. In Transportation Networks: Recent Methodological Advances (ed. M. G. H. Bell), pp. 163-174. Amsterdam:Pergamon Press.

Dafermos, S. \& Sparrow, F. T. 1969 The traffic assignment problem for a general network. Journal of Research of the National Bureau of Standards, 73B (1969), no. 1, pp. 91-118.

Daganzo, C. F. 1983 Stochastic network equilibrium with multiple vehicle types and asymmetric, indefinite link cost Jacobians. Transportation Science 17, no. 3, pp. 282-300.

Daganzo, C. F. \& Sheffi, Y. 1977 On stochastic models of traffic assignment. Transportation Science 11, no. 3 , pp. $253-274$.

Davis, G. A. 1994 Exact local solution of the continuous network design problem via stochastic user equilibrium assignment. Transportation Research Part B 28), no. 1, pp. 61-75.

De Wolf, D. \& Smeers, Y. 1997 A stochastic version of a Stackelberg-Nash-Cournot equilibrium model. Management Science 43, no. 2, pp. 190-197.

El Ghaoui, L. \& Lebret, H. 1997 Robust solutions to least-squares problems with uncertain data. SIAM Journal on Matrix Analysis and Applications 18, no. 4, pp. 1035-1064.

Eliasson, J. \& Mattsson, L.-G. 2006 Equity effects of congestion pricing: Quantitative methodology and a case study for Stockholm. Transportation Research Part A 40, no. 7, pp. 602-620.

Evgrafov, A. \& Patriksson, M. 2003a Stochastic structural topology optimization: Discretization and penalty function approach. Structural and Multidisciplinary Optimization 25, no. 3, pp. 174-188.

Evgrafov, A. \& Patriksson, M. 2003b Stable relaxations of stochastic stress constrained weight minimization problems. Structural and Multidisciplinary Optimization 25, no. 3, pp. 189-198.

Evgrafov, A. \& Patriksson, M. 2004 On the existence of solutions to stochastic mathematical programs with equilibrium constraints. Journal of Optimization Theory and Applications 121, no. 1, pp. 65-76.

Evgrafov, A. \& Patriksson, M. 2005 On the convergence of stationary sequences in topology optimization. International Journal of Numerical Methods in Engineering 64, no. 1, pp. 17-44.

Evgrafov, A., Patriksson, M. \& Petersson, J. 2002 On stochastic structural topology optimization. In Nonsmooth/Nonconvex Mechanics with Applications in Engineering (ed. C. C. Baniotopoulos), pp. 97104. Department of Civil Engineering, Faculty of Engineering, Aristotle University of Thessaloniki, Thessaloniki, Greece.

Evgrafov, A. Patriksson, M. \& Petersson, J. 2003 Stochastic structural topology optimization: Existence 
of solutions and sensitivity analyses. Zeitschrift für Angewandte Mathematik und Mechanik 83, no. 7, pp. 479-492.

Facchinei, F. \& Pang, J.-S. 2003a Finite-Dimensional Variational Inequalities and Complementarity Problems. Vol. I of Springer Series in Operations Research. Berlin: Springer-Verlag.

Facchinei, F. \& Pang, J.-S. 2003b Finite-Dimensional Variational Inequalities and Complementarity Problems. Vol. II of Springer Series in Operations Research. Berlin: Springer-Verlag.

Fisk, C. S. 1980 Some developments in equilibrium traffic assignment. Transportation Research Part B 14, no. 3, pp. 243-255.

Fletcher, R., Leyffer, S., Ralph, D. \& Scholtes, S. 2006 Local convergence of SQP methods for mathematical programs with equilibrium constraints. SIAM Journal on Optimization 17, no. 1, pp. 259-286.

Flyvbjerg, B., Holm, M. K. S. \& Buhl, S. L. 2005 How (in)accurate are demand forecasts in public works projects? The case of transportation. Journal of the American Planning Association 71, no. 1, pp. 131-146.

Friesz, T. L., Cho, H.-J., Metha, N. J., Tobin, R. L. \& Anandalingam, G. 1992 A simulated annealing approach to the network design problem with variational inequality constraints. Transportation Science 26, no. 1 , pp. $18-26$.

Fukushima, M. 1992 Equivalent differentiable optimization problems and descent methods for asymmetric variational inequality problems. Mathematical Programming, 53, no. 1, pp. 99-110.

Fukushima, M. \& Pang, J.-S. 1999 Convergence of a smoothing continuation method for mathematical programs with complementarity constraints. In Ill-Posed Variational Problems and Regularization Techniques (eds. M. Théra \& R. Tichatschke), pp. 99-110, vol. 477 of Lecture Notes in Economical and Mathematical Systems, Berlin: Springer-Verlag.

Guo, X., Cheng, G. \& Yamazaki, K. 2001. A new approach for the solution of singular optima in truss topology optimization with stress and local buckling constraints. Structural and Multidisciplinary Optimization 22, no. 5, pp. 364-372.

Gur, Y. J., Turnquist, M. A., Schneider, M., LeBlanc, L. J. \& Kurth, D. 1979 Estimation of an origindestination trip table based on observed link volumes and turning movements, volume 1: Technical report. Final Report DOT-FH-11-9292, U. S. Federal Highway Administration, Washington, D.C.

Gürkan, G., Özge, A. Y. \& Robinson, S. M. 1998 Sample-path solutions for simulation optimization problems and stochastic variational inequalities. In Interfaces in Computer Science and Operations Research (ed. D. W. Woodruff), pp. 169-188. Boston, MA; USA: Kluwer Academic Publishers.

Gürkan, G., Özge, A. Y. \& Robinson, S. M. 1999a Sample-path solution of stochastic variational inequalities. Mathematical Programming 84, no. 2, pp. 313-333.

Gürkan, G., Özge, A. Y. \& Robinson, S. M. 1999b Solving stochastic optimization problems with stochastic constraints: An application in network design. In Proceedings of the 31st Conference on Winter Simulation: Simulation $-A$ Bridge to the Future (eds. P. A. Farrington, H. B. Nembhard, D. T. Sturrock, \& G. W. Evans), pp. 471-478. New York, NY: ACM Press.

Gwinner, J. \& Raciti, F. 2006 Random equilibrium problems on networks. Mathematical and Computer Modelling 43, no. 7-8, pp. 880-891.

Heitsch, H., Römisch, W. \& Strugarek, C. 2006 Stability of multistage stochastic programs. SIAM Journal on Optimization 17, no. 2, pp. 511-525.

Henrion, R. \& Römisch, W. 2004 Hölder and Lipschitz stability of solution sets in programs with probabilistic constraints. Mathematical Programming 100, no. 3, pp. 589-611.

Himmelberg, C. J. 1975 Measurable relations. Fundamenta Mathematicae 87, no. 1, pp. 53-72.

Hobbs, B. F. \& Pang, J. S. 2007 Nash-Cournot equilibria in electric power markets with piecewise linear demand functions and joint constraints. Operations Research 55, no. 1, pp. 113-127.

Hu, X. \& Ralph, D. 2004 Convergence of a penalty method for mathematical programming with complementarity constraints. Journal of Optimization Theory and Applications 123, no. 2, pp. 365-390.

Josefsson, M. \& Patriksson, M. 2007 Sensitivity analysis of separable traffic equilibria, with application 
to bilevel optimization in network design. Transportation Research Part B 41, no 1, pp. 4-31.

Kall, P. \& Wallace, S. W. 1994 Stochastic Programming. Chichester, U.K.: John Wiley \& Sons. Freely downloadable at

http://www. unizh.ch/ior/Pages/Deutsch/Mitglieder/Kall/bib/ka-wal-94.pdf.

Karamardian, S. 1969a The nonlinear complementarity problem with applications, part 1. Journal of Optimization Theory and Applications 4, no. 1, pp. 87-98.

Karamardian, S. 1969b The nonlinear complementarity problem with applications, part 2. Journal of Optimization Theory and Applications 4, no. 1, pp. 167-181.

Kelly, F. 2006 Road pricing: Addressing congestion, pollution and the financing of Britain's roads. Ignenia 29, no. 1, pp. 34-40.

Kelly, F. Maulloo, A. \& Tan, D. 1998 Rate control for communication networks: Shadow prices, proportional fairness and stability. Journal of the Operational Research Society 49, no. 3, pp. 237-252.

Kelly, F. \& Voice, T. 2005 Stability of end-to-end algorithms for joint routing and rate control. Computer Communications Review 35, no. 1, pp. 5-12.

Kirsch, U. 1990 On singular topologies in optimum structural design. Structural Optimization 2, no. 3, pp. 133-142.

Kleywegt, A. J., Shapiro, A., \& Homem de Mello, T. 2001 The sample average approximation method for stochastic discrete optimization. SIAM Journal on Optimization 12, no. 2, pp. 479-502.

Larsson, T. \& Patriksson, M. 1994a A class of gap functions for variational inequalities. Mathematical Programming 64, no. 1, pp. 53-79.

Larsson, T. \& Patriksson, M. 1994b Equilibrium characterizations of solutions to side constrained asymmetric traffic assignment models. Le Matematiche 49, no. 2, pp. 249-280.

Larsson, T. \& Patriksson, M. 1998 Side constrained traffic equilibrium models-Traffic management through link tolls. In Equilibrium and Advanced Transportation Modelling (eds. P. Marcotte \& S. Nguyen), pp. 125-151. Boston, MA, USA: Kluwer Academic Publishers.

Larsson, T. \& Patriksson, M. 1999 Side constrained traffic equilibrium models: Analysis, computation and applications. Transportation Research Part B 33, no. 4, pp. 233-264.

Larsson, T., Patriksson, M. \& Rydergren, C. 2003 Inverse nonlinear multicommodity flow optimization by column generation. Optimization Methods and Software 18, no. 5, pp. 601-613.

Lawphongpanich, S. \& Hearn, D. W. 2004 An MPEC approach to second-best toll pricing. Mathematical Programming 101, no. 1, pp. 33-55.

LeBlanc, L. J., Morlok, E. K. \& Pierskalla, W. P. 1975 An efficient approach to solving the road network equilibrium traffic assignment problem. Transportation Research 9, no. 5, pp. 309-318.

Lepp, R. 1990 Approximations to stochastic programs with complete recourse, SIAM Journal on Control and Optimization 28, no. 2, pp. 382-394.

Lepp, R. 1992 Projection and discretization methods in stochastic programming, Journal of Computational and Applied Mathematics 56, no. 1, pp. 55-64.

Leyffer, S., López-Calva, G. \& Nocedal, J. 2007 Interior methods for mathematical programs with complementarity constraints. SIAM Journal on Optimization 17, no. 1, pp. 52-77.

Luo, Z.-Q., Pang, J.-S. \& Ralph, D. 1996 Mathematical Programs with Equilibrium Constraints. Cambridge, U.K.: Cambridge University Press.

Maher, M. J., Zhang, X. \& Van Vliet, D. 2001 A bi-level programming approach for trip matrix estimation and traffic control problems with stochastic user equilibrium link flows. Transportation Research Part B 35, no. 1, pp. 23-40.

Marcotte, P. 1986 Network design problem with congestion effects: A case of bilevel programming. Mathematical Programming 34, no. 2, pp. 142-146.

Marcotte, P. \& Marquis, G. 1992 Efficient implementation of heuristics for the continuous network design problem. Annals of Operations Research 34, 163-176. 
Marcotte, P. \& Patriksson, M. 2007 Traffic equilibrium. in Transportation (eds. C. Barnhart \& G. Laporte), pp. 623-713, vol. 14 of Handbooks in Operations Research and Management Science. Amsterdam: North-Holland.

Marti, K. (ed.) 1997 Structural reliability and stochastic structural optimization, special issue 3 of Mathematical Methods in Operations Research 46.

Meng, F. \& Xu, H. 2006 A regularized sample average approximation method for stochastic mathematical programs with nonsmooth equality constraints. SIAM Journal on Optimization 17, no. 3, pp. 891-919.

Meng, Q. \& Yang, H. 2002 Benefit distribution and equity in road network design. Transportation Research Part B 36, no. 1, pp. 19-35.

Meng, Q., Yang, H. \& Bell, M. G. H. 2001 An equivalent continuously differentiable model and a locally convergent algorithm for the continuous network design problem. Transportation Research Part B 35, no. 1, pp.83-105.

Mitra, D. \& Wang, Q. 2005 Stochastic traffic engineering for demand uncertainty and risk-aware network revenue management. IEEE/ACM Transactions on Networking 13, no. 2, pp. 221-233.

Olsen, P. 1976 Multistage stochastic programming with recourse as mathematical programming in an $l_{p}$ space. SIAM Journal on Control and Optimization 14, no. 3, pp. 528-537.

Outram, V. E. \& Thompson, E. 1978 Drivers perceived cost in route choice. In Proceedings of the PTRC Seminar on Transportation Models, pp. 226-257. University of Warwich, Warwich, U.K.

Outrata, J., Kočvara, M. \& Zowe, J. 1998 Nonsmooth Approach to Optimization Problems with Equilibrium Constraints. Dordrecht: Kluwer Academic Publishers.

Pang, J.-S. 1997 Error bounds in mathematical programming. Mathematical Programming 79, no. 1-3, pp. 299-332.

Patriksson, M. 1994 The Traffic Assignment Problem-Models and Methods. Topics in Transportation. Utrecht, The Netherlands: VSP BV. Freely downloadable at

http://www. math.chalmers.se/〜mipat/traffic.html.

Patriksson, M. 2004 Sensitivity analysis of traffic equilibria. Transportation Science 38, no. 3, pp. 258281.

Patriksson, M. 2008 Robust bilevel optimization models in transportation science. Philosophical Transactions of the Royal Society, A: Mathematical, Physical \& Engineering Sciences 366, no. 1872, pp. 19892004.

Patriksson, M. \& Petersson, J. 2002 Existence and continuity of optimal solutions to some structural topology optimization problems including unilateral constraints and stochastic loads. Zeitschrift für Angewandte Mathematik und Mechanik 82, no. 7, pp. 435-459.

Patriksson, M. \& Rockafellar, R. T. 2002 A mathematical model and descent algorithm for bilevel traffic management. Transportation Science 36, no. 3, pp. 271-291.

Patriksson, M. \& Rockafellar, R. T. 2003 Sensitivity analysis of variational inequalities over aggregated polyhedra, with application to traffic equilibria. Transportation Science 37, no. 1, pp. 56-68.

Patriksson, M. \& Wynter, L. 1999 Stochastic mathematical programs with equilibrium constraints. Operations Research Letters 25, no. 4, pp. 159-167.

Petersson, J. 2001 On continuity of the design-to-state mappings for trusses with variable topology. International Journal of Engineering Science 39, no. 10, pp. 1119-1141.

Plambeck, E. L., Fu, B.-R., Robinson, S. M. \& Suri, R. 1993 Throughput optimization in tandem production lines via nonsmooth programming. In Proceedings of the 1993 Summer computer simulation conference (ed. J. Schoen), pp. 70-75. San Diego, CA, USA: Society for Computer Simulation.

Plambeck, E. L., Fu, B.-R., Robinson, S. M. \& Suri, R. 1996 Sample-path optimization of convex stochastic performance functions. Mathematical Programming 75, no. 2, pp. 137-176.

Prékopa, A. 1995 Stochastic Programming. Dordrecht, The Netherlands: Kluwer Academic Publishers.

Prékopa, A. 2003 Probabilistic programming. In Stochastic programming (eds. A. Ruszczyński \& 
A. Shapiro), pp. 267-351, vol. 10 of Handbooks In Operations Research And Management Science. Amsterdam: Elsevier.

Ralph, D. 2008 Mathematical programs with complementarity constraints in traffic and telecommunications networks. Philosophical Transactions of the Royal Society, A: Mathematical, Physical \& Engineering Sciences, 366, no. 1872, pp. 1973-1987.

Robinson, S. M. 1980 Strongly regular generalized equations. Mathematics of Operations Research 5, no. 1 , pp. $43-62$.

Robinson, S. M. 1996 Analysis of sample-path optimization. Mathematics of Operations Research 21, no. 3 , pp. $513-528$.

Robinson, S. M. \& Wets, R. J.-B. 1987 Stability in two-stage stochastic programming. SIAM Journal on Control and Optimization 25, no. 6, pp. 1409-1416.

Rockafellar, R. T. \& Wets, R. J.-B. 1976 Stochastic convex programming: Relatively complete recourse and induced feasibility. SIAM Journal on Control and Optimization 14, no. 3, pp. 574-589.

Rockafellar, R. T. \& Wets, R. J.-B. 1998 Variational Analysis. Berlin, Germany: Springer-Verlag.

Rozvany, G. I. N. \& Birker, T. 1994 On singular topologies in exact layout optimization. Structural Optimization 8, no. 4, pp. 228-235.

Rubinstein, R. Y. \& Shapiro, A. 1993 Discrete Event Systems: Sensitivity Analysis and Stochastic Optimization by the Score Function Method. Chichester, U.K.: John Wiley \& Sons.

Römisch, W. 2003 Stability of stochastic programming problems. In Stochastic Programming (eds. A. Ruszczyński \& A. Shapiro), pp. 483-554, vol. 10 of Handbooks In Operations Research And Management Science. Amsterdam: Elsevier.

Römisch, W. \& Schultz, R. 1991 Distribution sensitivity in stochastic programming. Mathematical Programming 50, no. 2, pp. 197-226.

Scheel, H. \& Scholtes, S. 2000 Mathematical programs with complementarity constraints: Stationarity, optimality and sensitivity. Mathematics of Operations Research 25, no. 1, pp. 1-22.

Scholtes, S. 2001 Convergence properties of a regularization scheme for mathematical programs with complementarity constraints. SIAM Journal on Optimization 110, no. 4, pp. 918-936.

Scholtes, S. \& Stöhr, M. 1999 Exact penalization of mathematical programs with equilibrium constraints. SIAM Journal on Control and Optimization 37, no. 2, pp. 617-652.

Sender, J. G. \& Netter, M. 1970 Équilibre offre-demande et tarification sur un réseau de transport. Rapport de recherche 3, Département Economie, Institut de Recherche des Transports, Arcueil, France.

Shapiro, A. 2003 Monte Carlo sampling methods. In Stochastic Programming (eds. A. Ruszczyński \& A. Shapiro), pp. 353-425, vol. 10 of Handbooks In Operations Research And Management Science. Amsterdam: Elsevier.

Shapiro, A. 2006 Stochastic programming with equilibrium constraints. Journal of Optimization Theory and Applications 128, no. 1, pp. 223-243.

Shapiro, A. 2008 Stochastic programming approach to optimization under uncertainty. Mathematical Programming 112, no. 1, pp. 183-220.

Shapiro, A. \& Xu, H. 2005 Stochastic mathematical programs with equilibrium constraints, modeling and sample average approximation. Preprint, School of Industrial and Systems Engineering, Georgia Institute of Technology, Atlanta, GA, USA.

Sheffi, Y. 1985 Urban Transportation Networks: Equilibrium Analysis with Mathematical Programming Methods. Englewood Cliffs, NJ, USA: Prentice-Hall.

Soyster, A. L. 1973 Convex programming with set-inclusive constraints and applications to inexact linear programming. Operations Research 21, no. 5, pp. 1154-1157.

Stewart, K. 2007 Tolling traffic links under stochastic assignment: Modelling the relationships between the number and price level of tolled links and optimal traffic flows. Transportation Research Part A 41, no. 7 , pp. $644-654$. 
Stolpe, M. \& Svanberg, K. 2001 On trajectories of the epsilon-relaxation approach for stress constrained truss topology optimization. Structural and Multidisciplinary Optimization 21, no. 2, pp. 140-151.

Sumalee, A., Watling, D. P. \& Nakayama, S. 2006 Reliable network design problem: Case with uncertain demand and total travel time reliability. Transportation Research Record 1964, pp. 81-90.

U. S. Bureau of Public Roads 1964 Traffic assignment manual. U. S. Bureau of Public Roads, U. S. Government Printing Office, Washington, D.C.

Uchida, T. \& Iida, Y. 1993 Risk assignment: A new traffic assignment model considering the risk of travel time variation. In: Transportation and Traffic Theory, Proceedings of the 12th International Symposium on the Theory of Traffic Flow and Transportation, Berkeley, CA, July 21-23, 1993 (ed. C. F. Daganzo), pp. 89-105. Amsterdam, The Netherlands: Elsevier.

Ukkusuri, S. V., Mathew, T. V. \& Waller, S. T. 2007 Robust transportation network design under demand uncertainty. Computer-Aided Civil and Infrastructure Engineering 22, no. 1, pp. 6-18.

Ukkusuri, S. V \& Patil, G. R. 2006 An MPEC approach to stochastic transportation network design problem. Preprint, Department of Civil and Environmental Engineering, Renselaer Polytechnic Institute, Troy, NY, USA.

Vainikko, Y. 1971 The convergence of the method of mechanical quadratures for integral equations with discontinuous kernels. Akademija Nauk SSSR. Sibirskoe Otdelenie. Sibirskiı̌ Matematičeskiı̌ Žurnal 12, no. 1 , pp. $40-53$.

Van Slyke, R. M. \& Wets, R. J.-B. 1969 L-shaped linear programs with applications to optimal control and stochastic programming. SIAM Journal on Applied Mathematics 17, no. 4, pp. 638-663.

Von Falkenhausen, H. 1966 Traffic assignment by a stochastic model. In Proceedings of the 4th International Conference on Operational Science, pp. 415-421.

Wallace, S. W. 2000 Decision making under uncertainty: Is sensitivity analysis of any use? Operations Research, 48, no. 1, pp. 20-25.

Wardrop, J. G. 1952 Some theoretical aspects of road traffic research. Proceedings of the Institute of Civil Engineers, Part II, 325-378.

Wynter, L. 2002 Stochastic bilevel programs. In Encyclopedia of Optimization (eds. P. A. Floudas \& P. M. Pardalos), vol. V (R-Z), pp. 290-297. New York, NY, USA: Kluwer Academic Publishers.

$\mathrm{Xu}, \mathrm{H}$. 2005 An MPCC approach for stochastic Stackelberg-Nash-Cournot equilibrium. Optimization 54, no. 1 , pp. $27-57$.

Yang, H. 1995 Heuristic algorithms for the bilevel origin-destination matrix estimation problems. Transportation Research Part B 29, no. 4, pp. 231-242.

Yang, H., Iida, Y. \& Sasaki, T. 1991 An analysis of the reliability of an origin-destination trip matrix estimated from traffic counts. Transportation Research Part B 25, no. 5, pp. 351-363.

Yang, H., Xu, W. \& Weng, Q. 2005 A sequential experimental approach for analyzing second-best road pricing with unknown demand functions. In Flow, Dynamics and Human Interaction, Proceedings of the 16th International Symposium on Transportation and Traffic Theory (ed. H. S. Mahmassani), pp. 23-42. Amsterdam, The Netherlands: Elsevier.

Ye, J. J., Zhu, D. L. \& Zhu, Q. J. 1997 Exact penalization and necessary optimality conditions for generalized bilevel programming problems. SIAM Journal on Optimization 7, no. 2, pp. 481-507.

Yen, N. D. 1995 Lipschitz continuity of solutions of variational inequalities with a parametric polyhedral constraint. Mathematics of Operations Research 20, no. 3, pp. 695-708.

Zhang, R. 1994 Problems of hierarchical optimization in finite dimension. SIAM Journal on Optimization 40, no. 3, pp. 521-536. 